\title{
NORMALITY AND QUADRATICITY FOR SPECIAL AMPLE LINE BUNDLES ON TORIC VARIETIES ARISING FROM ROOT SYSTEMS
}

\author{
QËNDRIM R. GASHI \\ Department of Mathematics, University of Prishtina, Pristina 10000, Kosovo \\ e-mail:qendrim@gmail.com \\ and TRAVIS SCHEDLER \\ Department of Mathematics, The University of Texas at Austin 2515 Speedway, Austin, TX 78712-1202, USA \\ e-mail: trasched@gmail.com
}

\begin{abstract}
We prove that special ample line bundles on toric varieties arising from root systems are projectively normal. Here the maximal cones of the fans correspond to the Weyl chambers, and special means that the bundle is torus-equivariant such that the character of the line bundle that corresponds to a maximal Weyl chamber is dominant with respect to that chamber. Moreover, we prove that the associated semi-group rings are quadratic.
\end{abstract}

2010 Mathematics Subject Classification. 17B22, 14M25.

\section{Introduction and statement of main results.}

1.1. Notation. Let $\Phi$ be an irreducible, reduced root system of rank $n$. We write $Y, X$, $X^{\vee}$ and $Y^{\vee}$ for the root, weight, co-root, and co-weight lattice, respectively. Note that $Y \subseteq X$ and $X^{\vee} \subseteq Y^{\vee}$. We also write $\Phi$ for the corresponding set of roots of the root system.

Denote by $W$ the Weyl group of $\Phi$. Let $F$ be the Weyl fan in $Y^{\vee} \otimes_{\mathbb{Z}} \mathbb{R}$ and $F_{n} \subset F$ be the set of chambers (i.e. cones of maximal dimension) in $F$. The elements of $F_{n}$ are the Weyl chambers cut out by the root hyperplanes of $\Phi$.

We study the complex toric variety $V$, whose fan is $F$, co-character lattice is $N:=Y^{\vee}$ and character lattice is $M:=Y$. The toric variety $V$ has been studied by many authors, e.g. $[3,4,5,14,17,20,24]$. It is a smooth, projective toric variety for the torus $T_{1}=\operatorname{Spec}(\mathbb{C}[Y]) \simeq\left(\mathbb{C}^{\times}\right)^{n}$. It is a well-known fact (although we do not use it) that those toric varieties are closures of generic torus orbits in the flag variety $G / B$ where $G$ is the reductive group associated to $\Phi$ and $B \subseteq G$ is a Borel subgroup.

Since $T_{1}$ acts on $V$, the torus $T=\operatorname{Spec}(\mathbb{C}[X])$ also acts on $V$ via the canonical projection $T \rightarrow T_{1}$. Let $\mathcal{L}$ be a $T$-equivariant ample line bundle on $V$. Such line bundles (or, more precisely, the isomorphism classes thereof) are in one-to-one correspondence (see, e.g. [13]) with convex polytopes $P \subset X \otimes_{\mathbb{Z}} \mathbb{R}=M \otimes_{\mathbb{Z}} \mathbb{R}$ satisfying the following property: The vertices of $P$ are given by a set $\left\{\mu_{\sigma}: \sigma \in F_{n}\right\} \subset X$, and for any two vertices $\mu_{\sigma}$ and $\mu_{\sigma^{\prime}}$ of $P$, where $\sigma$ and $\sigma^{\prime}$ are adjacent chambers, $\mu_{\sigma}-\mu_{\sigma^{\prime}}=r_{\sigma, \sigma^{\prime}} \alpha_{\sigma, \sigma^{\prime}}$, for some number $r_{\sigma, \sigma^{\prime}} \in \mathbb{Z}_{>0}$, where $\alpha_{\sigma, \sigma^{\prime}} \in \Phi$ is the unique root that is positive on $\sigma$ and negative on $\sigma^{\prime}$. Such polytopes are called 'ample'.(In, e.g. [1], the sets $\left\{\mu_{\sigma}\right\}$ are called 'strictly positive orthogonal sets' in this case.) Here we use the negative notation of [13]: what we call $P$ corresponds to $-P$ there. 
We denote by $\Lambda(P)$ the set of points $x \in P \cap X$ whose image in $X / Y$ coincides with the images in $X / Y$ of the vertices of $P$, i.e. $\Lambda(P)=P \cap\left\{y+\mu_{\sigma} \mid y \in Y\right\}$ for any choice of Weyl chamber $\sigma$. Note that the character $x \in X$ occurs in $H^{0}(X, \mathcal{L})$ if and only if $x \in \Lambda(P)$, where $P$ is the polytope corresponding to $\mathcal{L}$ (and then it occurs with multiplicity one); see, e.g. [21, Section 23.1, p. 496].

To every chamber $\sigma \in F_{n}$ there corresponds a basis $\left\{\alpha_{i, \sigma}: i \in I\right\} \subseteq \Phi$ of $Y$ consisting of elements of $\Phi$, where $I:=\{1, \ldots, n\}$ (in other words, a choice of simple roots). More precisely, once and for all we fix a set of simple roots which is dominant for a fixed chamber, say $\sigma_{0}$, and then obtain all of these bases by the action of the Weyl group so that $\alpha_{i, w \cdot \sigma_{0}}=w \cdot \alpha_{i, \sigma_{0}}$ for all $w \in W$. Here we say that an element $x \in X \otimes_{\mathbb{Z}} \mathbb{R}$ is $\sigma$-dominant (or dominant for $\sigma$ ) if, for all $y \in \sigma,\langle x, y\rangle \geq 0$. The pairing $\langle-,-\rangle$ here is the $\mathbb{R}$-bilinear extension of the usual pairing $X \times X^{\vee} \rightarrow \mathbb{Z}$, and the element $\alpha_{i, \sigma}^{\vee}$ is the co-root of $\Phi$ corresponding to the root $\alpha_{i, \sigma}$. We can conclude from the definition of $\alpha_{i, \sigma}$ that $x$ is $\sigma$-dominant if and only if $\left\langle x, \alpha_{i, \sigma}^{\vee}\right\rangle \geq 0$ for all $i$.

We impose a restriction on the type of polytopes $P$ that we consider, which is essential to our method of proof:

(†) For every $\sigma \in F_{n}$, the element $\mu_{\sigma}$ is $\sigma$-dominant.

Following Kottwitz ([21, Section 12.9, p. 44]), we call ample polytopes satisfying the property $(\dagger)$ special. ${ }^{1}$ In what follows, we will primarily be interested in special ample polytopes. Note that the Weil divisors of such ample polytopes, in particular, must have strictly positive coefficients of all prime $T$-invariant divisors (but this condition does not imply speciality).

The condition to be special says that all of the vertices of the polytope lie in their corresponding Weyl chambers; geometrically this roughly says that the polytope is 'not too thin in any root direction'. Precisely, we give below examples of special and nonspecial polytopes in the case of the root system $A_{2}$. Denote the simple roots by $\alpha$ and $\beta$ and the simple co-roots by $\alpha^{\vee}$ and $\beta^{\vee}$. Thus, the set of positive roots is $\{\alpha, \beta, \alpha+\beta\}$, and the set of positive co-roots is $\left\{\alpha^{\vee}, \beta^{\vee}, \alpha^{\vee}+\beta^{\vee}\right\}$.

EXAMPLE 1.1. Two special polytopes for the root system $A_{2}$ are as follows. These differ only by shifting (i.e., they define the same non-equivariant bundle). Note that most shifts of this polytope are not special: we require that each vertex corresponding to a given chamber remain in that chamber, or on its boundary (the second example has vertices on the boundary of their chambers). Note that, by ampleness, we are already restricted to hexagons whose sides are perpendicular to the axes drawn.

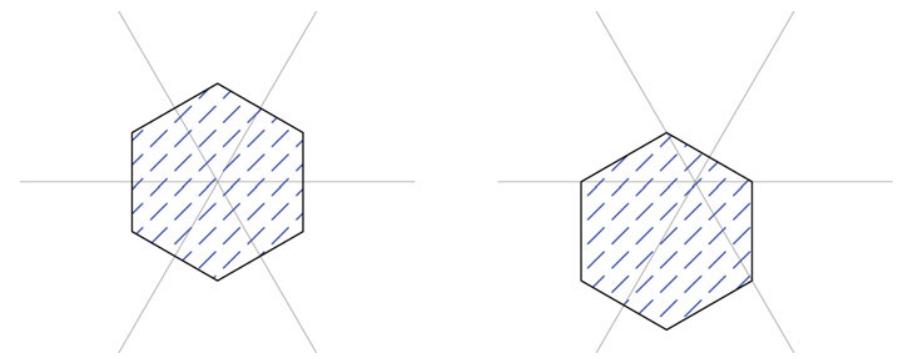

\footnotetext{
${ }^{1}$ More generally, Kottwitz [21] defines special orthogonal sets, where an orthogonal set is a collection $\left\{\mu_{\sigma}\right\}$ where $\mu_{\sigma}-\mu_{\sigma}^{\prime}=r_{\sigma, \sigma^{\prime}} \alpha_{\sigma, \sigma^{\prime}}$ for $r_{\sigma, \sigma^{\prime}} \in \mathbb{Z}$, not necessarily positive. A special orthogonal set is then one satisfying property $(\dagger)$. They necessarily satisfy $r_{\sigma, \sigma^{\prime}} \geq 0$ for all adjacent $\sigma, \sigma^{\prime}$ (i.e. they are 'positive orthogonal sets'), but the $r_{\sigma, \sigma^{\prime}}$ need not be positive (i.e. $\left\{\mu_{\sigma}\right\}$ need not be strictly positive, as in the ample case). The associated divisors are in particular a non-negative linear combination of prime $T$-invariant divisors.
} 
EXAMPLE 1.2. Another special polytope, which is not symmetric under the Weyl group (after a shift), is given by

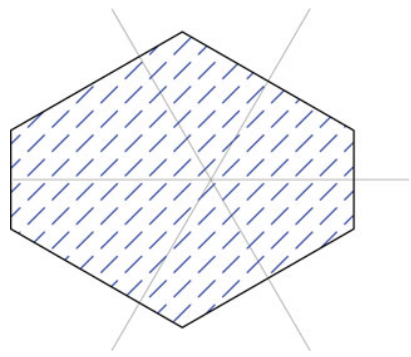

EXAMPLE 1.3. Any of the above special ample polytopes can be easily made nonspecial by shifting in a direction so that the vertices are no longer in the appropriate chambers. For example, one such non-special polytope is:

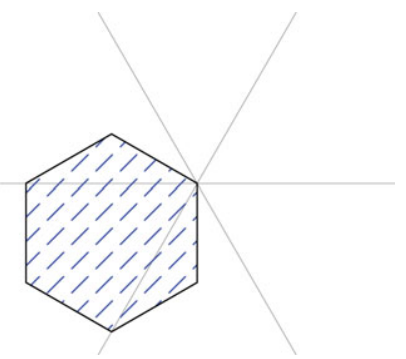

EXAMPLE 1.4. An example of a non-special ample polytope for the root system $A_{2}$ which cannot be shifted to obtain a special ample polytope is as follows:

1.2. Statement of main results. Our first main result is the following, which will be proved in Section 2.

THEOREM 1.5. Let $P$ be a special ample polytope as above and let $m \in \mathbb{Z}_{>0}$. Consider the dilated polytope $m P:=\{m x: x \in P\}$. Then any point $z \in \Lambda(m P)$ can be written as $a$ sum $z=z_{1}+\cdots+z_{m}$, with $z_{i} \in \Lambda(P), \forall i=1, \ldots, m$.

The toric interpretation of the theorem is as follows. Call an equivariant line bundle $\mathcal{L}$ on $V$ special ample if it corresponds to a special ample polytope $P$. 
COROLlaRY 1.6. Let $\mathcal{L}$ be a special ample line bundle on $V$. Then the canonical map

$$
H^{0}(V, \mathcal{L}) \otimes H^{0}(V, \mathcal{L}) \otimes \cdots \otimes H^{0}(V, \mathcal{L}) \longrightarrow H^{0}\left(V, \mathcal{L}^{m}\right)
$$

is a surjection for all $m \geq 1$, i.e. $\mathcal{L}$ is projectively normal.

REMARK 1.7. The above corollary is a special case of Oda's Conjecture which claims that the statement of the corollary is true for any ample line bundle on a nonsingular, projective toric variety. In the case of root systems of type $A$, the conjecture, and therefore the corollary, is known to be true (see [19]).

Next, consider the semi-group $S_{P} \subset X \times \mathbb{Z}$ generated by $(x, 1)$ for $x \in \Lambda(P)$. Then the main theorem is equivalent to the statement that $S_{P}$ is normal, i.e. it is saturated in $X \times \mathbb{Z}$. In other words, it equals its saturation, $\overline{S_{P}}:=\bigcup_{m \geq 1}(\Lambda(m P)) \times\{m\}$, i.e. the intersection of the cone $\mathbb{R}_{>0} \cdot(P \times\{1\})$ with the lattice $\left\{\left(y+t \mu_{\sigma}, t\right) \mid y \in Y, t \in \mathbb{Z}\right\}$ for any fixed $\sigma \in F_{n}$.

If we instead begin with the semi-group $\overline{S_{P}}$, then Theorem 1.5 is equivalent to the statement that this semi-group is generated in degree one with respect to the grading $\operatorname{deg}(x, m)=m$, for $x \in X$ and $m \in \mathbb{Z}$.

Our second main result is as follows.

THEOREM 1.8. Let $P$ be special ample. Then the semi-group $S_{P}=\overline{S_{P}}$ is presented by quadratic relations. In other words, $S_{P}=\langle\Lambda(P) \times\{1\}\rangle /(R)$, where $R$ is spanned by the elements

$$
(x, 1)(y, 1)-\left(x^{\prime}, 1\right)\left(y^{\prime}, 1\right)
$$

for $x, y, x^{\prime}, y^{\prime} \in \Lambda(P)$ such that $x+y=x^{\prime}+y^{\prime}$.

REMARK 1.9. Put differently, the semi-group ring $\mathbb{C}\left[S_{P}\right]=\mathbb{C}\left[\overline{S_{P}}\right]$ is quadratic. We will actually prove a stronger version of the above theorem, which roughly says that, when $P$ is special, $(R)$ is spanned by moves which replace $\left(x_{1}, \ldots, x_{m}\right) \in \Lambda(P)^{m}$ by $\left(x_{1}, \ldots, x_{i}+\alpha, x_{i+1}-\alpha, \ldots, x_{m}\right)$ for $\alpha \in \Phi$. See Section 1.3 for a precise statement.

Since $\mathbb{C}\left[\overline{S_{P}}\right] \cong \bigoplus_{m \geq 0} H^{0}\left(V, \mathcal{L}^{m}\right)$, the toric interpretation of Theorems 1.5 and 1.8 is as follows.

COROLlary 1.10. Let $\mathcal{L}$ be a special ample line bundle on $V$. Then the ring $\bigoplus_{m \geq 0} H^{0}\left(V, \mathcal{L}^{m}\right)$ is quadratic.

REMARK 1.11. The above corollary is a special case of Sturmfels's conjecture [29, Conjecture 13.19], which states that, for any projective non-singular toric variety $X$ and ample projectively normal line bundle $\mathcal{L}$, the associated ring $\bigoplus_{m \geq 0} H^{0}\left(X, \mathcal{L}^{m}\right)$ is quadratic. (If Oda's conjecture is true, then the projectively normal assumption is automatic.)

This leaves open the natural

QUeSTION 1.12. Is the ring $\mathbb{C}\left[S_{P}\right]$ Koszul?

See Sections 1.4 and 6 for such a result in a related situation.

REMARK 1.13. It is clear that all of the above results remain true if we replace a special ample polytope $P$ with the polytope $v+P$, where $v \in X$ (we still require that 
$P$ satisfies property $(\dagger))$. Geometrically, the corresponding line bundles are isomorphic as non-equivariant bundles, which explains why the results continue to hold.

1.3. Strengthening Theorems 1.5 and 1.8. Rather than prove Theorem 1.8, we will prove the following, which generalizes it and Theorem 1.5. For yet another strengthening, see Appendix A.

Definition 1.14. Suppose $P_{1}, \ldots, P_{m}$ are special ample polytopes and $\left(x_{1}, \ldots, x_{m}\right) \in \Lambda\left(P_{1}\right) \times \cdots \times \Lambda\left(P_{m}\right)$. Further suppose that $\beta \in \Phi$ is a root and $i$ and $j$ are indices such that $x_{i}+\beta \in \Lambda\left(P_{i}\right)$ and $x_{j}-\beta \in \Lambda\left(P_{j}\right)$. Then we say that

$$
\left(x_{1}, \ldots, x_{m}\right) \sim\left(x_{1}, \ldots, x_{i-1}, x_{i}+\beta, x_{i+1}, \ldots, x_{j-1}, x_{j}-\beta, x_{j+1}, \ldots, x_{m}\right) .
$$

Call this a root move. Extend $\sim$ to the equivalence relation generated by this, i.e. $\left(x_{1}, \ldots, x_{m}\right) \sim\left(x_{1}^{\prime}, \ldots, x_{m}^{\prime}\right)$ if the two are related by a sequence of root moves.

Note that, since root moves are reversible, a tuple is related to another tuple by root moves if and only if one can be obtained from the other by a sequence of root moves.

The following result strengthens Theorems 1.5 and 1.8.

THeOREM 1.16. If $P_{1}, \ldots, P_{m}$ are special ample polytopes and $x \in \Lambda\left(P_{1}+\cdots+\right.$ $\left.P_{m}\right)$, then

(i) there exists a tuple $\left(x_{1}, \ldots, x_{m}\right) \in \Lambda\left(P_{1}\right) \times \cdots \times \Lambda\left(P_{m}\right)$ such that $x_{1}+\cdots+$ $x_{m}=x$;

(ii) if $\left(x_{1}, \ldots, x_{m}\right)$ and $\left(x_{1}^{\prime}, \ldots, x_{m}^{\prime}\right)$ are two such tuples, then $\left(x_{1}, \ldots, x_{m}\right) \sim$ $\left(x_{1}^{\prime}, \ldots, x_{m}^{\prime}\right)$.

Specializing to the case $m=2$ and $P=P_{1}=P_{2}$, part (ii) implies that the permutation $\left(x_{1}, x_{2}\right) \mapsto\left(x_{2}, x_{1}\right)$ is a series of root moves inside $\Lambda(P)^{2}$. Therefore, in the case $P=P_{1}=\cdots=P_{m}$ for arbitrary $m$, the relation $\sim$ is actually generated by root moves (1.15) with $j=i+1$. This explains Remark 1.9, and hence Theorem 1.16.(ii) implies Theorem 1.8 .

Our motivation for allowing $P_{1}, \ldots, P_{m}$ to be distinct polytopes is that it allows one to inductively prove the theorem on $m$ : one deduces the result for $m>2$ from the pair $\left(P_{1}+\cdots+P_{m-1}, P_{m}\right)$.

A toric interpretation of part of the theorem is as follows. Let $\mathcal{L}_{1}, \ldots, \mathcal{L}_{m}$ be special ample line bundles on $V$ and

$$
\varphi_{\mathcal{L}_{1}, \ldots, \mathcal{L}_{m}}: H^{0}\left(V, \mathcal{L}_{1}\right) \otimes \cdots \otimes H^{0}\left(V, \mathcal{L}_{m}\right) \longrightarrow H^{0}\left(V, \mathcal{L}_{1} \otimes \cdots \otimes \mathcal{L}_{m}\right)
$$

be the canonical map.

COROllary 1.17 .

(i) $\varphi_{\mathcal{L}_{1}, \ldots, \mathcal{L}_{m}}$ is surjective.

(ii) The kernel of $\varphi_{\mathcal{L}_{1}, \ldots, \mathcal{L}_{m}}$ is spanned by the canonical subspaces

$$
\operatorname{ker}\left(\varphi_{\mathcal{L}_{i}, \mathcal{L}_{j}}\right) \otimes \bigotimes_{k \notin\{i, j\}} H^{0}\left(V, \mathcal{L}_{k}\right) \subseteq \operatorname{ker}\left(\varphi_{\mathcal{L}_{1}, \ldots, \mathcal{L}_{m}}\right)
$$

Namely, Theorem 1.16.(i) implies Corollary 1.17.(i), since if $P_{i}$ are the polytopes such that $\Lambda\left(P_{i}\right)$ is a basis of $H^{0}\left(V, \mathcal{L}_{i}\right)$ for all $i$, then $P_{1}+\cdots+P_{m}$ is the polytope 
such that $\Lambda\left(P_{1}+\cdots+P_{m}\right)$ is the basis of $H^{0}\left(V, \mathcal{L}_{1} \otimes \cdots \otimes \mathcal{L}_{m}\right)$. Similarly, Theorem 1.16.(ii) implies Corollary 1.17.(ii) since the moves generating the equivalence relation $\sim$ are multiples of quadratic relations in $S_{P_{1}+\cdots+P_{m}}$ of the form $t^{x_{i}} t^{x_{j}}=t^{x_{i}+\beta} t^{x_{j}-\beta}$, for $x_{i}, x_{i}+\beta \in \Lambda\left(P_{i}\right), x_{j}, x_{j}-\beta \in \Lambda\left(P_{j}\right)$, and $\beta \in \Phi$ a root, where for $x_{i} \in \Lambda\left(P_{i}\right)$ we denote by $t^{x_{i}}$ the corresponding basis element of $H^{0}\left(V, \mathcal{L}_{i}\right)$.

Similarly, we can apply this to the Cayley sum polytope of polytopes $P_{1}, \ldots, P_{k}$. Recall that this is defined as the polytope inside $\left(X \otimes_{\mathbb{Z}} \mathbb{R}\right) \times \mathbb{R}^{k}$, which is the convex hull of $\left(P_{1} \times\left\{e_{1}\right\}\right) \cup \cdots \cup\left(P_{k} \times\left\{e_{k}\right\}\right)$, where $e_{1}, \ldots, e_{k}$ are the standard basis of $\mathbb{R}^{k}$. The resulting polytope is denoted by $P_{1} * P_{2} * \cdots * P_{k}$ and is considered with respect to the lattice $Y \times \mathbb{Z}^{k}$.

Corollary $1.18 .^{2}$ Let $P_{1}, \ldots, P_{k}$ be special ample polytopes. Then the Cayley sum polytope $P=P_{1} * \cdots * P_{k}$ is normal, and $\mathbb{C}\left[S_{P}\right]$ is quadratic.

The corollary follows from Theorem 1.16 as follows: For every $m_{1}, \ldots, m_{k} \geq 0$, apply the theorem to the product $\Lambda\left(P_{1}\right)^{m_{1}} \times \cdots \times \Lambda\left(P_{k}\right)^{m_{k}}$, with $m=m_{1}+\cdots+m_{k}$. Note here that the (degree-one) generators of $\mathbb{C}\left[S_{P}\right]$ are the elements $\left(\left(y, e_{i}\right), 1\right) \in$ $\left(\Lambda\left(P_{i}\right) \times \mathbb{Z}^{k}\right) \times \mathbb{Z}$, where $1 \leq i \leq k$.

Finally, we give the toric interpretation of the corollary. Let $\mathcal{L}_{1}, \ldots, \mathcal{L}_{k}$ be special ample line bundles on $V$. Given a vector bundle $\mathcal{U}$, let $\operatorname{Sym}^{m}(\mathcal{U})$ denote its $m$ th symmetric power.

Corollary 1.19. The ring $\bigoplus_{m \geq 0} H^{0}\left(V, \operatorname{Sym}^{m}\left(\mathcal{L}_{1} \oplus \cdots \oplus \mathcal{L}_{k}\right)\right)$ is quadratic.

1.4. Diagonal splitness. A closely related toric variety to $V$, studied in, e.g. [23], is the one whose fan is such that its rays (i.e. one-dimensional cones) are generated by the elements of $\Phi$ : so its initial lattice is $Y$, dual to the initial lattice of $V$. Denote this variety by $U$.

Suppose that $Q$ is an orthogonal polytope corresponding to the fan associated to $U$, i.e. one which describes an equivariant line bundle on $U$. Then in [23] it was proved that $Q$ is always diagonally split (see [23] or Section 6). This property was intended to prove Koszulity (and in particular normality and quadraticity) of $\mathbb{C}\left[S_{Q}\right]$, although since the publication of Payne's paper [23] (and the submission of the present one) a counterexample (the $3 \times 3$ Birkhoff polytope) to [23, Theorem 1.4] was found.

Nonetheless, we explain in Section 6 that, unlike in the situation of [23], the ample polytopes $P$ associated to the toric variety $V$ considered in this paper are not diagonally split except in the cases $A_{1}, A_{2}, A_{3}$ and $B_{2}$.

1.5. Organization of the paper. In Section 2 we prove Theorem 1.5, where a crucial step involves using a lemma of Stembridge ([28, Cor. 2.7]) stating that in the usual partial order of $\sigma$-dominant weights, a weight $v$ covers another one $v^{\prime}$ if and only if the difference $v-v^{\prime}$ is a root that is positive with respect to $\sigma$. In Section 3 we recall the numbers game with a cutoff (from [15]; see also [18]), which gives a useful language to prove Theorem 1.16. The proof of the theorem is then given in Sections 4 and 5 . Note that one of our auxiliary results (Lemma 4.4) generalizes the above lemma of Stembridge. In Section 6 we show that ample polytopes for the toric varieties $V$ as above are not diagonally split (with the exception of the cases when the root system $\Phi$ is of type $A_{1}, A_{2}, A_{3}$ or $B_{2}$ ).

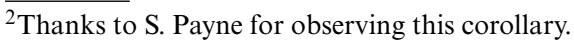


In Appendix A, we give a generalization of Theorem 1.16 in terms of the numbers game: these allow one to restrict the type of tuples needed in the equivalence $\sim$ above.

2. Proof of Theorem 1.5. Recall that for a cone $\sigma \in F_{n}$, we denote by $\left\{\alpha_{i, \sigma}: i \in\right.$ I\} $\subset \Phi$ the corresponding set of simple roots. For a root $\gamma \in \Phi$, we say that it is positive or negative with respect to the chamber $\sigma$ if $\gamma$ can be written as a non-negative or nonpositive linear combination of the elements of $\left\{\alpha_{i, \sigma}: i \in I\right\}$ respectively. We write $D_{\sigma}$ for the set of $\sigma$-dominant elements of $X \otimes_{\mathbb{Z}} \mathbb{R}$.

Note that $P$ is the convex hull of the points $\left\{\mu_{\sigma} \in X: \sigma \in F_{n}\right\}$. The next two lemmas will allow us to better understand the shape of the polytope $P$. The first one uses the fact that $P$ is ample and the second one that $P$ is special.

LeMma 2.1. (see, e.g. [21, Lemma 12.1, p. 445]).

$$
P=\bigcap_{\sigma \in F_{n}} C_{\sigma}^{*},
$$

where $C_{\sigma}^{*}:=\left\{\mu_{\sigma}-\sum_{i=1}^{n} t_{i} \alpha_{i, \sigma}: t_{i} \in \mathbb{R}_{\geq 0}\right\}$.

LeMma 2.2. (see, e.g. [21, Lemma 12.2, p. 445]).

$$
P \cap D_{\sigma}=C_{\sigma}^{*} \cap D_{\sigma}
$$

Specializing to the points in $\Lambda(P)$, we obtain

$$
\Lambda(P) \cap D_{\sigma}=\left\{v \in D_{\sigma} \cap X: v \stackrel{\sigma}{\preceq} \mu_{\sigma}\right\},
$$

where $\stackrel{\sigma}{\preceq}$ stands for the partial order in $X$ determined by the chamber $\sigma$, i.e. $v \stackrel{\sigma}{\leq} \mu_{\sigma}$ if $\mu_{\sigma}-v$ is a non-negative integral linear combination of the roots $\left\{\alpha_{i, \sigma}: i \in I\right\}$.

Fix a chamber $\sigma \in F_{n}$. Since $W$ acts simply transitively on $\left\{D_{\tau}: \tau \in F_{n}\right\}$ and since $\Lambda(m P)=\bigcup_{w \in W}\left(\Lambda(m P) \cap\left(w D_{\sigma}\right)\right)$, it suffices to prove the statement of Theorem 1.5 for $z \in \Lambda(m P) \cap D_{\sigma}$.

By (2.3), every element $z \in \Lambda(m P) \cap D_{\sigma}$ satisfies $z \stackrel{\sigma}{\preceq} m \mu_{\sigma}$. Clearly, for $z=m \mu_{\sigma}$ the assertion of Theorem 1.5 is true. So to prove the theorem, it suffices to show that, whenever it holds for $x \in \Lambda(m P) \cap D_{\sigma}$, then it also holds for every $z \in \Lambda(m P) \cap D_{\sigma}$ such that $x$ covers $z$. Here $x$ covers $z$ means that $z \stackrel{\sigma}{\preceq} t \stackrel{\sigma}{\preceq} x$ and $t \in D_{\sigma} \cap X$ implies that $t=z$ or $t=x$.

So assume that the statement of the theorem is true for $x \in \Lambda(m P) \cap D_{\sigma}$ and that $x$ covers $z \in \Lambda(m P) \cap D_{\sigma}$. By a lemma of Stembridge ([28, Cor. 2.7]; see also [27, Lemma 2.3] and Remark 4.5), there exists a $\sigma$-positive $\operatorname{root} \beta$ such that $x-z=\beta$. Since $z$ is $\sigma$-dominant and $\beta$ is $\sigma$-positive, $\left\langle z, \beta^{\vee}\right\rangle \geq 0$, and thus $\left\langle x-\beta, \beta^{\vee}\right\rangle \geq 0$, i.e.

$$
\left\langle x, \beta^{\vee}\right\rangle \geq 2
$$

By assumption, $x$ can be written as a sum $x=x_{1}+\cdots+x_{m}$, with $x_{i} \in \Lambda(P), \forall i=$ $1, \ldots, m$. The last inequality guarantees that $\left\langle x_{j}, \beta^{\vee}\right\rangle \geq 1$ for at least one $j \in\{1, \ldots, m\}$. The proposition below then ensures that $x_{j}-\beta \in P$. 
Proposition 2.4. Let $y \in \Lambda(P)$ and $\beta \in \Phi$. If $\left\langle y, \beta^{\vee}\right\rangle \geq 1$, then $y-\beta \in \Lambda(P)$.

We now put $z_{i}=x_{i}, \forall i \neq j$, and $z_{j}=x_{j}-\beta$, and then $z=z_{1}+\cdots+z_{m}$, which verifies the theorem. This concludes the proof of Theorem 1.5, and it only remains to prove the last proposition.

2.1. Proof of Proposition 2.4. Let $y \in \Lambda(P)$ and $\beta \in \Phi$ be such that $\left\langle y, \beta^{\vee}\right\rangle \geq 1$.

We must show that, for every $\sigma \in F_{n}, y-\beta \stackrel{\sigma}{\preceq} \mu_{\sigma}$. Note that, since $y \in \Lambda(P)$, there exist non-negative integers $h_{i, \sigma}, i=1, \ldots, n$, such that

$$
\mu_{\sigma}-y=\sum_{i=1}^{n} h_{i, \sigma} \alpha_{i, \sigma}
$$

Let $\beta=\sum_{i=1}^{n} b_{i, \sigma} \alpha_{i, \sigma}$. Then $\mu_{\sigma}-(y-\beta)=\sum_{i=1}^{n}\left(h_{i, \sigma}+b_{i, \sigma}\right) \alpha_{i, \sigma}$. If the chamber $\sigma$ is such that $\beta$ is positive with respect to it, then clearly $y-\beta \stackrel{\sigma}{\preceq} \mu_{\sigma}$.

We are therefore left to consider only the chambers with respect to which $\beta$ is negative (recall that $\beta$ must be positive or negative with respect to each chamber). Denote the set of such chambers by $F_{-}$. Then we can write $F_{-}$as a disjoint union $F_{-}=F_{-}^{\prime} \cup F_{-}^{\prime \prime}$, where

$F_{-}^{\prime}=\left\{\tau \in F_{-}: \tau\right.$ is adjacent to a chamber with respect to which $\beta$ is positive $\}$,

and $F_{-}^{\prime \prime}=F_{-} \backslash F_{-}^{\prime}$.

Since $P$ is special, $\left\langle\mu_{\sigma}, \beta^{\vee}\right\rangle \leq 0, \forall \sigma \in F_{-}$. Moreover, we claim that $\left\langle\mu_{\sigma}, \beta^{\vee}\right\rangle \leq$ $-1, \forall \sigma \in F_{-}^{\prime \prime}$. This follows from the previous statement because $P$ is ample: indeed, if $\sigma \in F_{-}^{\prime \prime}$, then by definition $-\beta$ is positive but not simple for $\sigma$, so there exists $\alpha_{i, \sigma}$ (necessarily not equal to $-\beta$ ) such that $\left\langle\alpha_{i, \sigma},-\beta^{\vee}\right\rangle \geq 1$. Therefore, if $\left\langle\mu_{\sigma}, \beta^{\vee}\right\rangle=0$, then if $\sigma^{\prime}$ is the chamber adjacent to $\sigma$ corresponding to $\alpha_{i, \sigma}$, it follows that $\left\langle\mu_{\sigma^{\prime}}, \beta^{\vee}\right\rangle=\left\langle\mu_{\sigma}-r_{\sigma, \sigma^{\prime}} \alpha_{i, \sigma}, \beta^{\vee}\right\rangle \geq r_{\sigma, \sigma^{\prime}}>0$. However, $\sigma^{\prime} \in F_{-}$by definition, which furnishes a contradiction.

To prove the proposition, we claim that it suffices to show that $y-\beta \stackrel{\sigma}{\leq} \mu_{\sigma}$ when $\sigma \in F_{-}^{\prime}$. Since $\left\langle y-\beta, \beta^{\vee}\right\rangle \geq-1$ by assumption, it will then follow that $y-\beta$ lies in all of the half-spaces whose intersection defines $P$ (whose boundaries are maximaldimensional facets of $P$ ), except possibly for those whose boundary planes meet vertices of $P$ only in $F_{-}^{\prime \prime}$. Suppose, for the sake of contradiction, that $y-\beta \notin P$. Let $0 \leq t<1$ be maximal such that $y-t \beta \in P$. Then $y-t \beta$ lies on a boundary plane meeting vertices of $P$ only in $F_{-}^{\prime \prime}$. Since $\left\langle\mu_{\sigma}, \beta^{\vee}\right\rangle \leq-1, \forall \sigma \in F_{-}^{\prime \prime}$, it follows that $\left\langle y-\beta, \beta^{\vee}\right\rangle<$ $\left\langle y-t \beta, \beta^{\vee}\right\rangle \leq-1$. This is impossible, since $\left\langle y, \beta^{\vee}\right\rangle \geq 1$. Thus, $y-\beta \in P$, as desired.

Thus, take $\sigma \in F_{-}^{\prime}$. In the remainder of the proof, we show that $y-\beta \stackrel{\sigma}{\preceq} \mu_{\sigma}$. Denote by $\tau$ a chamber in $F$ that is adjacent to $\sigma$ and such that $\beta$ is positive with respect to $\tau$. We write $\alpha_{i}$ instead of $\alpha_{i, \tau}$ for the simple roots corresponding to $\tau$. Since $\beta$ is negative with respect to $\sigma$, there exists $j \in I$ such that $\beta=\alpha_{j}=-\alpha_{j, \sigma}$. Moreover,

$$
\alpha_{i}=\alpha_{i, \sigma}+\left\langle\alpha_{i}, \beta^{\vee}\right\rangle \beta
$$


Since $P$ is ample, $\mu_{\sigma}=-r_{\tau, \sigma} \beta+\mu_{\tau}$, for $r_{\tau, \sigma}>0$. Thus, using (2.5) and applying (2.6), we get

$$
\sum_{i=1}^{n} h_{i, \sigma} \alpha_{i, \sigma}=-r_{\tau, \sigma} \beta+\sum_{i=1}^{n} h_{i, \tau}\left(\alpha_{i, \sigma}+\left\langle\alpha_{i}, \beta^{\vee}\right\rangle \beta\right) .
$$

Since $\left\{\alpha_{i, \sigma}: i \in I\right\}$ is the basis for $\Phi$ and $\alpha_{j, \sigma}=-\beta$, from the last identity we deduce that

$$
\begin{gathered}
h_{i, \sigma}=h_{i, \tau}, \forall i \in I \backslash\{j\}, \quad \text { and } \\
h_{j, \sigma}=r_{\tau, \sigma}-h_{j, \tau}-\sum_{i \in I \backslash\{j\}} h_{i, \tau}\left\langle\alpha_{i}, \beta^{\vee}\right\rangle .
\end{gathered}
$$

Now $\mu_{\sigma}-(y-\beta)=\left(\sum_{i=1}^{n} h_{i, \sigma} \alpha_{i, \sigma}\right)-\alpha_{j, \sigma}$, so in order to prove that $y-\beta \stackrel{\sigma}{\preceq} \mu_{\sigma}$, it suffices to show that $h_{j, \sigma} \geq 1$. For a contradiction, assume $h_{j, \sigma}=0$. From (2.7) we then get

$$
h_{j, \tau}-r_{\tau, \sigma}=-\sum_{i \in I \backslash\{j\}} h_{i, \tau}\left\langle\alpha_{i}, \beta^{\vee}\right\rangle .
$$

Next, recall that $\left\langle y, \beta^{\vee}\right\rangle \geq 1$, so

$$
\begin{gathered}
\left\langle\mu_{\sigma}, \beta^{\vee}\right\rangle=\left\langle\mu_{\tau}-r_{\tau, \sigma} \beta, \beta^{\vee}\right\rangle=\left\langle y+\sum_{i=1}^{n} h_{i, \tau} \alpha_{i}, \beta^{\vee}\right\rangle-2 r_{\tau, \sigma} \\
\geq 1+2 h_{j, \tau}-2 r_{\tau, \sigma}+\sum_{i \in I \backslash j j\}} h_{i, \tau}\left\langle\alpha_{i}, \beta^{\vee}\right\rangle=1-\sum_{i \in I \backslash\{j\}} h_{i, \tau}\left\langle\alpha_{i}, \beta^{\vee}\right\rangle,
\end{gathered}
$$

where to get the last equality we used (2.8). But the last expression is strictly positive (since $\left\langle\alpha_{i}, \beta^{\vee}\right\rangle=\left\langle\alpha_{i}, \alpha_{j}^{\vee}\right\rangle \leq 0$ for all $i \neq j$ ), and, since the polytope $P$ is special, $\left\langle\mu_{\sigma}, \alpha_{j}^{\vee}\right\rangle \leq 0$, a contradiction. This ends the proof that $y-\beta \stackrel{\sigma}{\preceq} \mu_{\sigma}$ and concludes the proof of Proposition 2.4.

3. The numbers game with a cutoff. In order to prove Theorem 1.8 , we use the language of the numbers game with a cutoff, from [16] (see also [18]). In this section we recall what we will need.

3.1. The usual numbers game. We first recall Mozes's numbers game [22], which has been widely studied in, e.g. $[6,7,8,9,10,11,12,25,26,30,31])$. Fix an unoriented, finite graph with no loops and no multiple edges. Let $I$ be the set of vertices. Fix also a Cartan matrix $C=\left(c_{i j}\right)_{i, j \in I} \in \mathbb{R}^{I} \times \mathbb{R}^{I}$ such that $c_{i i}=2$ for all $i, c_{i j}=0$ whenever $i$ and $j$ are not adjacent, and otherwise $c_{i j}, c_{j i}<0$, and either $c_{i j} c_{j i}=4 \cos ^{2}\left(\frac{\pi}{n_{i j}}\right)$ for $n_{i j} \geq 1$ integers or $c_{i j} c_{j i} \geq 4$.

We will only need to consider the case where our graph is the underlying graph of a Dynkin diagram (undirected and without multiple edges), and $C$ is the standard Cartan matrix for the diagram, i.e. $c_{i j}=\left\langle\alpha_{i}, \alpha_{j}^{\vee}\right\rangle$. In particular, $c_{i j} \in \mathbb{Z}$ for all $i, j$. Hence, the reader may assume this if desired. 
The configurations of the game consist of vectors from $\mathbb{R}^{I}$. The moves of the game are as follows: For any vector $v \in \mathbb{R}^{I}$ and any vertex $i \in I$ such that $v_{i}<0$, one may perform the following move, called firing the vertex $i$ : $v$ is replaced by the new configuration $f_{i}(v)$, defined by

$$
f_{i}(v)_{j}=v_{j}-c_{i j} v_{i}
$$

The entries $v_{i}$ of the vector $v$ are called amplitudes. The game terminates if all the amplitudes are non-negative. Let us emphasize that only negative-amplitude vertices may be fired. ${ }^{3}$

PROPOSITION 3.1. [12]. The numbers game is strongly convergent: If the game can terminate, then it must terminate, and in exactly the same number of moves and arriving at the same configuration, regardless of the choices made.

3.2. The numbers game with a cutoff. In [15], the numbers game with a cutoff was defined: The moves are the same as in the ordinary numbers game, but the game continues (and in fact starts) only as long as all amplitudes remain greater than or equal to -1 . Such configurations are called allowed. Every configuration which does not have this property is called forbidden, and upon reaching such a configuration the game terminates (we lose). We call a configuration winning if it is possible, by playing the numbers game with a cutoff, to reach a configuration with all non-negative amplitudes.

In [18], a simple criterion was given to determine when the numbers game with a cutoff is winning. We will restrict to the Dynkin case, with $C$ the standard Cartan matrix. Let $\Phi$ be the set of roots. Pick simple roots corresponding to the vertices of the Dynkin diagram, and write $\Phi=\Phi_{+} \sqcup-\Phi_{+}$, where $\Phi_{+}$is the set of positive roots.

We can view $\Phi \subseteq \mathbb{Z}^{I}$ and $\Phi_{+} \subseteq \mathbb{Z}_{\geq 0}^{I}$. For $i \in I$, let $\alpha_{i}$ be the simple root corresponding to $i$, which as an element of $\mathbb{Z}^{I}$ is the elementary vector $\left(\alpha_{i}\right)_{j}=\delta_{i j}$. Note that, since $\alpha_{i}$ refers to a vector in $\mathbb{Z}^{I}$, in the case that $\alpha \in \mathbb{Z}^{I}$, we will never use $\alpha_{i}$ to refer to a component of $\alpha$, reserving it exclusively for the elementary vector $\alpha_{i} \in \mathbb{Z}^{I}$.

A useful description of $\Phi$ is

$$
\Phi=\bigcup_{i \in I} W \cdot \alpha_{i},
$$

where $W$ is the Weyl group generated by the simple reflections $s_{i}: \mathbb{R}^{I} \rightarrow \mathbb{R}^{I}$, for all $i \in I$, given by

$$
s_{i}(\beta)_{j}=\left\{\begin{array}{ll}
\beta_{j}, & \text { if } j \neq i \\
-\beta_{i}-\sum_{k \neq i} c_{k i} \beta_{k}, & \text { if } j=i
\end{array} .\right.
$$

Proposition 3.2. [18, Theorem 3.1, Corollary 5.10.(a)]. Fix a Dynkin diagram with standard Cartan matrix $C$. Beginning with a configuration $v \in \mathbb{R}^{I}$, the numbers game with a cutoff is winning if and only if

$$
v \cdot \alpha \geq-1, \forall \alpha \in \Phi_{+},
$$

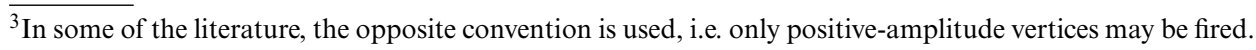


and in this case, one always wins the numbers game with a cutoff, no matter which moves are made, and arrives at the same final configuration in the same total number of moves. $c_{i} \in \mathbb{R}$.

Here, $\cdot$ is the dot product of $v \in \mathbb{R}^{I}$ with $\alpha \in \mathbb{Z}^{I}$, i.e. $v \cdot\left(\sum_{i} c_{i} \alpha_{i}\right)=\sum_{i} c_{i} v_{i}$, for

3.3. Relation to the polytope $P$. Proposition 2.4 has the following consequence in terms of the numbers game with a cutoff. We consider the embedding

$$
\iota: X \hookrightarrow \mathbb{R}^{I}, \quad x \mapsto \iota(x), \quad \iota(x)_{i}:=\left\langle x, \alpha_{i}^{\vee}\right\rangle .
$$

In this language, condition (3.3) translates for $x \in X$ as follows: The configuration $\iota(x)$ is winning if and only if

$$
\left\langle x, \alpha^{\vee}\right\rangle \geq-1, \forall \alpha \in \Phi_{+} .
$$

Then Proposition 2.4 implies the following.

COROLlaRY 3.5. If $x, y \in X$ and $\iota(y)$ can be obtained from $\iota(x)$ by playing the numbers game with a cutoff, then $x \in \Lambda(P)$ if and only if $y \in \Lambda(P)$.

Proof. Suppose that $u \in X$ and $\iota(u) \in \mathbb{Z}^{I}$ is obtained along the way from $\iota(x)$ to $\iota(y)$. From $u$, any move in the numbers game with a cutoff is of the form $u \mapsto u+\iota\left(\alpha_{i}\right)$ for some $i \in I$ such that $u_{i}=-1$. Hence, $\left\langle u, \alpha_{i}^{\vee}\right\rangle=-1$ and $\left(u+\iota\left(\alpha_{i}\right)\right)_{i}=\left\langle u+\alpha_{i}, \alpha_{i}^{\vee}\right\rangle=1$. We therefore conclude from Proposition 2.4 that $u \in \Lambda(P)$ if and only if $u+\alpha_{i} \in \Lambda(P)$. The corollary follows.

Note that the choice of simple roots was arbitrary, so the corollary in fact holds for any choice of simple roots (equivalently, any choice of dominant chamber).

REMARK 3.6. The corollary extends to the case where $y$ is obtained from $x$ in the usual numbers game by firing vertices only of amplitude -1 , i.e. we can continue the numbers game even if there is an amplitude less than -1 as long as we never fire such vertices. (This seems to be a reasonable variation on the numbers game with a cutoff.)

4. Proof of Theorem 1.16. It is convenient to abuse notation slightly, by omitting the map $\iota$ :

NotATion 4.1. If $x \in X$ and $\iota(x)$ is winning, we also say that $x$ is winning. Moreover, if $x, y \in X$ and $\iota(y)$ is obtained from $\iota(x)$ by playing the numbers game (with or without a cutoff), we also say that $y$ is obtained from $x$ by playing the numbers game (with or without a cutoff respectively).

Fix once and for all a dominant chamber $\sigma$, and write $D, \prec, \preceq$, and $\mu$, instead of $D_{\sigma}, \stackrel{\sigma}{\prec}, \stackrel{\sigma}{\preceq}$, and $\mu_{\sigma}$, respectively. We omit $\sigma$ from now on, and by a dominant element we always mean an element of $D$.

Next, given special ample polytopes $P_{1}, \ldots, P_{m}$, we let $\mu_{1}, \ldots, \mu_{m}$ denote the vertices $\mu_{1}, \ldots, \mu_{m} \in D$ of each corresponding to the dominant chamber.

Finally, we recall the notion of length of roots. For simply laced root systems (i.e. types $A_{n}, D_{n}$ and $E_{n}$, since we only consider the Dynkin case), we say that all roots have the same length. For the other root systems, the set of roots $\Phi$ is partitioned into the subsets of short and long roots, and we say that the long roots are longer than the 
short roots. One way to define the partition (which will be useful to us) is that, if $\beta \in \Phi$ is at least as long as $\alpha \in \Phi$ and $\alpha \neq \pm \beta$, then $\left\langle\alpha, \beta^{\vee}\right\rangle \in\{-1,0,1\}$. Recall also that the partition is preserved by the Weyl group action. We emphasize that, for us, $\left\langle\alpha, \alpha^{\vee}\right\rangle=2$ for all $\alpha \in \Phi$, long or short; the terminology of length comes from the norm under the symmetrized Cartan form, which we will not use.

4.1. Outline of the proof. First, Theorem 1.16.(i) follows in exactly the same manner as Theorem 1.5. We give a short proof in the spirit of this section, based on Proposition 2.4 , in Section 4.4.

Our strategy underlying the proof of Theorem 1.16.(ii) is to perform induction on the sum $x_{1}+\cdots+x_{m}$, which we can assume is winning (in fact, we could assume it is dominant using the action of the Weyl group). The induction will be over a certain partial order on the sum polytope $P=P_{1}+\cdots+P_{m}$.

The proof is broken into three parts: First we prove results about the partial order on the winning locus of $P$, which boil down to the strengthening of the lemma of Stembridge mentioned earlier. Second, we prove the theorem in the case $m=2$. Third, we inductively deduce the theorem for general $m$. In what follows, we will explain the proof modulo some lemmas whose proofs will be provided in Section 5.

4.2. Partial ordering on the winning locus of $P$. Let $P$ be a special ample polytope.

Definition 4.2. Suppose $x \in \Lambda(P)$. If $x \neq \mu$, then a simple root $\alpha$ is $P$-progressive for $x$ if either $x$ is dominant and $\alpha$ has minimum length such that $x+\alpha \preceq \mu$, or else $\left\langle x, \alpha^{\vee}\right\rangle \leq-1$. for $x$.

It is immediate that, for all $x \neq \mu$, there exists a simple root which is $P$-progressive

This subsection is devoted to the proof of the following.

Proposition 4.3. If $\alpha$ is P-progressive for $x$, then $x+\alpha \in \Lambda(P)$. Moreover, if $x$ is winning, so is $x+\alpha$.

Proof. First suppose that $\alpha$ is a simple root such that $\left\langle x, \alpha^{\vee}\right\rangle \leq-1$. By Proposition 2.4, $x+\alpha \in \Lambda(P)$. If $x$ is winning, then $x+\alpha$ is obtained from $x$ by a move of the numbers game, and hence it is winning.

If $x$ is dominant and $\alpha$ is a simple root of minimum length such that $x+\alpha \preceq \mu$, the result follows from the case $y=\mu$ of the Lemma 4.4. Namely, by Corollary 3.5, to show that $x+\alpha \in \Lambda(P)$, it suffices to show that $z \in \Lambda(P)$, where $z$ is the result of playing the numbers game with a cutoff beginning with $x+\alpha$. Next, if $\beta \in \Phi_{+}$is any positive root such that $x+\beta \preceq \mu$, then $\beta$ must be at least as long as $\alpha$; otherwise $\beta$ would be short and $\alpha$ long, and there would exist a short simple root $\gamma$ such that $\gamma \preceq \beta$. In the latter case, $x+\gamma \preceq x+\beta \preceq \mu$, contradicting our assumption that $\alpha$ has minimum length such that $x+\alpha \preceq \mu$. Therefore, we may apply Lemma 4.4 with $y=\mu$. We conclude that $x+\alpha$ is winning, i.e. $z$ is dominant, and also $z \preceq \mu$. Then, $z \in \Lambda(P)$ by (2.3).

Lemma 4.4. Suppose $x \prec y$ and $x, y \in X \cap D$. Let $\alpha \in \Phi_{+}$be a positive root of minimum length such that $x+\alpha \preceq y$. Then $x+\alpha$ is winning, and if $z$ is the result of playing the numbers game with a cutoff, then $x+\alpha \preceq z \preceq y$.

The lemma will be proved in Section 5.1. 
REMARK 4.5. Lemma 4.4 strengthens the aforementioned result of Stembridge. Specifically, if $y$ covers $x$, then $y=z$, i.e. $y$ is obtainable from $x+\alpha$ by playing the numbers game with a cutoff. In this case, $y=x+\beta$, where $\beta \in \Phi_{+}$is obtained from $\alpha$ by playing the numbers game (using the same firing sequence as for $x+\alpha \mapsto x+\beta$, which involves firing only vertices of amplitude -1 ). This was our motivation for replacing $\mu$ by $y$ in the statement of the lemma.

4.3. The case $m=2$ of Theorem 1.16.(ii). The heart of the proof of Theorem 1.16.(ii) is contained in the case $m=2$. Then general $m$ will be a straightforward generalization. In turn, the case $m=2$ is based on the following lemma.

Lemma 4.6. Let $P_{1}$ and $P_{2}$ be special ample polytopes, $\left(x_{1}, x_{2}\right) \in \Lambda\left(P_{1}\right) \times \Lambda\left(P_{2}\right)$, $P=P_{1}+P_{2}$, and $x=x_{1}+x_{2} \in \Lambda(P)$. If $\alpha$ is $P$-progressive for $x$, then there exists $\left(x_{1}^{\prime}, x_{2}^{\prime}\right)$ and an index $i \in\{1,2\}$ such that $\left(x_{1}, x_{2}\right) \sim\left(x_{1}^{\prime}, x_{2}^{\prime}\right)$ and $\alpha$ is $P_{i}$-progressive for $x_{i}^{\prime}$.

This will be proved in Section 5.2. Here we explain how it implies Theorem 1.16.(ii) in the case $m=2$.

Proof of Theorem 1.16.(ii) for $m=2$. As remarked earlier, it is enough to prove the theorem in the case that $x$ is winning (in fact, it is enough to prove the theorem in the case $x$ is dominant, by applying a suitable element of the Weyl group simultaneously to $x$ as well as to all of the $x_{i}$ ). Let $\mu=\mu_{1}+\mu_{2}$ (where by convention $\mu_{i}$ is the vertex of $P_{i}$ corresponding to the dominant chamber). The theorem is immediate in the case that $x=\mu$, since $x=x_{1}+x_{2}$ implies that $x_{1}=\mu_{1}$ and $x_{2}=\mu_{2}$ (and vice versa). Inductively, suppose that $x \in \Lambda(P)$ is winning, and for some $P$-progressive $\alpha$, the theorem holds for $x+\alpha$.

Suppose that $\left(x_{1}, x_{2}\right),\left(x_{1}^{\prime}, x_{2}^{\prime}\right) \in \Lambda\left(P_{1}\right) \times \Lambda\left(P_{2}\right)$ are pairs such that $x_{1}+x_{2}=x=$ $x_{1}^{\prime}+x_{2}^{\prime}$. Let $\alpha$ be $P$-progressive for $x$. By Lemma 4.6 (applied to both $\left(x_{1}, x_{2}\right)$ and $\left(x_{1}^{\prime}, x_{2}^{\prime}\right)$ separately), it is enough to assume that there exist indices $i$ and $j$ such that $\alpha$ is $P_{i}$-progressive for $x_{i}$ and $P_{j}$-progressive for $x_{j}^{\prime}$. Without loss of generality, suppose that $i=1$. Let $\left(y_{1}, y_{2}\right)$ and $\left(y_{1}^{\prime}, y_{2}^{\prime}\right)$ be given by $y_{1}=x_{1}+\alpha, y_{2}=x_{2}, y_{j}^{\prime}=x_{j}^{\prime}+\alpha$ and $y_{k}^{\prime}=x_{k}^{\prime}$ for $k \neq j$. Since $x_{1}+x_{2}+\alpha=x+\alpha=x_{1}^{\prime}+x_{2}^{\prime}+\alpha$, by hypothesis, $\left(y_{1}, y_{2}\right) \sim$ $\left(y_{1}^{\prime}, y_{2}^{\prime}\right)$. By induction on the number of root moves (1.15) required to realize the latter equivalence, Lemma 4.7 then implies that either $\left(x_{1}, x_{2}\right) \sim\left(y_{1}^{\prime}-\alpha, y_{2}^{\prime}\right) \in \Lambda\left(P_{1}\right) \times$ $\Lambda\left(P_{2}\right)$ or $\left(x_{1}, x_{2}\right) \sim\left(y_{1}^{\prime}, y_{2}^{\prime}-\alpha\right) \in \Lambda\left(P_{1}\right) \times \Lambda\left(P_{2}\right)$ (where, for the purposes of induction, we drop the assumption that $\alpha$ is $P_{1}$-progressive for $x_{1}$ and assume only that $x_{1}+\alpha \in$ $\Lambda\left(P_{1}\right)$, and similarly for $x_{j}^{\prime}$ and $\left.P_{j}\right)$. If the result is $\left(x_{1}^{\prime}, x_{2}^{\prime}\right)$, we are done. If not, the result must be $\left(x_{1}^{\prime} \pm \alpha, x_{2}^{\prime} \mp \alpha\right)$, which is related to $\left(x_{1}^{\prime}, x_{2}^{\prime}\right)$ by a single root move.

Above we needed the following lemma, whose proof will be given in Section 5.3.

LemmA 4.7. Suppose that $\left(x_{1}, x_{2}\right) \in \Lambda\left(P_{1}\right) \times \Lambda\left(P_{2}\right)$, and $\alpha$ is a simple root such that $x=x_{1}+x_{2}$ satisfies $\left\langle x, \alpha^{\vee}\right\rangle \geq-1$, and such that $x_{1}+\alpha \in \Lambda\left(P_{1}\right)$. If $\beta \in \Phi$ is such that $\left(x_{1}+\alpha+\beta, x_{2}-\beta\right) \in \Lambda\left(P_{1}\right) \times \Lambda\left(P_{2}\right)$, then either $\left(x_{1}+\beta, x_{2}-\beta\right) \in \Lambda\left(P_{1}\right) \times \Lambda\left(P_{2}\right)$ or $\left(x_{1}+\alpha+\beta, x_{2}-\alpha-\beta\right) \in \Lambda\left(P_{1}\right) \times \Lambda\left(P_{2}\right)$. In the latter case, either $\alpha+\beta \in \Phi$, or $\left(x_{1}+\alpha, x_{2}-\alpha\right) \in \Lambda\left(P_{1}\right) \times \Lambda\left(P_{2}\right)$.

4.4. Proof of Theorem $\mathbf{1 . 1 6}$ for general $m$. Let $\mu=\mu_{1}+\cdots+\mu_{m}$, where $\mu_{i}$ is the vertex of $P_{i}$ corresponding to the dominant chamber. The theorem is immediate in the 
case $x=\mu$. It is enough to prove the theorem when $x$ is winning under the inductive hypothesis that the theorem holds for $x+\alpha$ where $\alpha$ is $P$-progressive for $x$.

To prove part (i), suppose that $\left(y_{1}, \ldots, y_{m}\right) \in \Lambda\left(P_{1}\right) \times \cdots \times \Lambda\left(P_{m}\right)$ with $y_{1}+\cdots$ $+y_{m}=x+\alpha$. Then for some index $i,\left\langle y_{i}, \alpha^{\vee}\right\rangle \geq 1$, and by Proposition 2.4, $\left(y_{1}, \ldots, y_{i-1}, y_{i}-\alpha, y_{i+1}, \ldots, y_{m}\right) \in \Lambda\left(P_{1}\right) \times \cdots \times \Lambda\left(P_{m}\right)$, with the desired sum $x$.

For part (ii), we will additionally induct on $m$, i.e. we assume that the theorem holds for smaller values of $m$. Let $Q:=P_{1}+\cdots+P_{m-1}$ so that $P=Q+P_{m}$. Let $y=x_{1}+\cdots+x_{m-1}$ and $y^{\prime}=x_{1}^{\prime}+\cdots+x_{m-1}^{\prime}$. Then, by the previous section, there exist root moves relating $\left(y, x_{m}\right)$ to $\left(y^{\prime}, x_{m}^{\prime}\right)$. To turn this into root moves relating $\left(x_{1}, \ldots, x_{m}\right)$ and $\left(x_{1}^{\prime}, \ldots, x_{m}^{\prime}\right)$, it is enough to apply the theorem for the case $m-1$ (i.e. for $\left.\left(P_{1}, \ldots, P_{m-1}\right)\right)$ together with the following lemma.

Lemma 4.8. Suppose that $y \in Q=P_{1}+\cdots+P_{m-1}, \beta \in \Phi$ and $y+\beta \in Q$. Assume Theorem 1.16. $(i)$ holds for $\left(P_{1}, \ldots, P_{m-1}\right)$. Then there exists a tuple $\left(y_{1}, \ldots, y_{m-1}\right) \in$ $\Lambda\left(P_{1}\right) \times \cdots \times \Lambda\left(P_{m-1}\right)$ such that $y=y_{1}+\cdots+y_{m-1}$ and an index $j$ such that $y_{j}+\beta \in$ $\Lambda\left(P_{j}\right)$

The lemma will be proved in Section 5.4.

\section{Proof of lemmas.}

5.1. Proof of Lemma 4.4. We will use the following general result.

Claim 5.1. If $x \in \mathbb{Z}^{I}$ is dominant and $\alpha \in \Phi_{+}$is any positive root, and the usual numbers game on $x+\alpha$ does not involve firing any vertices corresponding to simple roots shorter than $\alpha$, then $x+\alpha$ is winning.

In particular, if $\alpha$ is a short positive root and $x \in \mathbb{Z}^{I}$ is dominant, then $x+\alpha$ is winning (equivalently, all short positive roots are themselves winning).

Proof. Let us play the usual numbers game on $x+\alpha$. If we fire a vertex $i$ corresponding to a simple root $\beta$ whose length is at least that of $\alpha$, then since $\left\langle\alpha, \beta^{\vee}\right\rangle \geq-1$, the amplitude at vertex $i$ is -1 . Since the length of $\alpha+\beta$ is equal to that of $\alpha$, we can replace $\alpha$ with $\alpha+\beta$, and then $x+(\alpha+\beta)$ takes one fewer move under the numbers game to reach a dominant configuration. By induction on the number of moves required to play the numbers game on $x+\alpha$, we see that all vertices fired have amplitude -1 , and hence $x+\alpha$ is winning.

Suppose that $y \in X \cap D$ and $\alpha$ is a positive root of minimum length such that $x+\alpha \preceq y$. Let us play the numbers game with a cutoff on $x+\alpha$. We claim that this only involves firing vertices corresponding to simple roots of length of at least $\alpha$. Then, by Claim 5.1, $x+\alpha$ is winning. Moreover, the result $z$ of playing the numbers game with a cutoff is the dominant configuration obtained from $x+\alpha$ by adding the minimum positive combination of simple roots. Since $y$ is dominant and $x+\alpha \preceq y, y$ is also such a configuration, and it follows that $z \preceq y$.

It remains to show that playing the numbers game beginning with $x+\alpha$ does not involve firing a vertex corresponding to a simple root of length shorter than $\alpha$. For a contradiction, suppose not, and consider the first vertex fired corresponding to a shorter simple root. Call this simple root $\gamma$. It follows as above that every dominant configuration is obtainable from $x+\alpha$ by adding simple roots adds $\gamma$ as well. Therefore, 
since $x+\alpha \preceq y$ and $y$ is dominant, also $x+\alpha+\gamma \preceq y$ and hence $x+\gamma \preceq y$, which is a contradiction.

5.2. Proof of Lemma 4.6. First, if $x$ is not dominant, then $\left\langle x, \alpha^{\vee}\right\rangle \leq-1$, and hence $\left\langle x_{i}, \alpha^{\vee}\right\rangle \leq-1$ for some $i$, which shows that $\alpha$ is $P_{i}$-progressive for $x_{i}$. So we can restrict to the dominant case. Thus, $\alpha$ has minimal length among simple roots such that $x+\alpha \preceq \mu$.

Given a simple root $\alpha$, let $P^{\alpha}$ denote the maximum-dimensional boundary facet of $P$ meeting $\mu$ which is parallel to the span of all simple roots other than $\alpha$. In other words (using Lemma 2.1), $x \in \Lambda\left(P^{\alpha}\right)$ if and only if $x \in \Lambda(P)$ but $x+\alpha \npreceq \mu$.

CLAIM 5.2. If any element $x_{i}$ of the pair $\left(x_{1}, x_{2}\right)$ is dominant, then either $x_{i} \in \Lambda\left(P_{i}^{\alpha}\right)$, or else $\alpha$ is $P_{i}$-progressive for $x_{i}$.

Proof. If $x_{i} \notin \Lambda\left(P_{i}^{\alpha}\right)$, then $\alpha$ must be of minimal length with this property, since $x_{i} \notin \Lambda\left(P_{i}^{\beta}\right)$ implies that $x_{1}+x_{2} \notin \Lambda\left(P^{\beta}\right)$, which implies by assumption that $\beta$ is at least as long as $\alpha$.

Now we prove the lemma. If, for any simple $\operatorname{root} \beta,\left\langle x_{1}, \beta^{\vee}\right\rangle \leq-1$ but $\left\langle x_{2}, \beta^{\vee}\right\rangle \geq 1$, we can perform a move $\left(x_{1}, x_{2}\right) \mapsto\left(x_{1}+\beta, x_{2}-\beta\right)$. So after performing such moves, we can assume that this does not happen. Since $x=x_{1}+x_{2}$ is dominant, this implies that $x_{1}$ is dominant. By Claim 5.2, we are done unless $x_{1} \in P_{1}^{\alpha}$. So assume this is the case. By performing moves of the form $\left(x_{1}, x_{2}\right) \mapsto\left(x_{1}-\beta, x_{2}+\beta\right)$ for simple roots $\beta \neq \alpha$ (which may make $x_{1}$ no longer dominant, but preserves the property that $\left.x_{1} \in P_{1}^{\alpha}\right)$, we can assume that $\left\langle x_{2}, \beta^{\vee}\right\rangle \geq 0$ for all simple roots $\beta \neq \alpha$, without changing the assumption that $x_{1} \in P_{1}^{\alpha}$. Then either $\left\langle x_{2}, \alpha^{\vee}\right\rangle \leq-1$, or $x_{2}$ is dominant. In the former case, $\alpha$ is $P_{2}$-progressive for $x_{2}$, as desired. In the latter case, by Claim 5.2, it is enough to suppose that $x_{2} \in P_{2}^{\alpha}$. However, in this case, $x=x_{1}+x_{2} \in P_{1}^{\alpha}+P_{2}^{\alpha}=P^{\alpha}$, contradicting our assumption that $x+\alpha \preceq \mu$.

5.3. Proof of Lemma 4.7. First, if $\left\langle x_{1}+\alpha+\beta, \alpha^{\vee}\right\rangle \geq 1$, then $x_{1}+\beta \in \Lambda\left(P_{1}\right)$ by Proposition 2.4. Since $x_{2}-\beta \in \Lambda\left(P_{2}\right)$ by assumption, this proves the lemma. Next, suppose that $\left\langle x_{1}+\alpha+\beta, \alpha^{\vee}\right\rangle \leq 0$, i.e. $\left\langle x_{1}+\beta, \alpha^{\vee}\right\rangle \leq-2$. Since $x=\left(x_{1}+\right.$ $\beta)+\left(x_{2}-\beta\right)$ satisfies $\left\langle x, \alpha^{\vee}\right\rangle \geq-1$, it follows that $\left\langle x_{2}-\beta, \alpha^{\vee}\right\rangle \geq 1$. By Proposition 2.4, $x_{2}-\beta-\alpha \in \Lambda\left(P_{2}\right)$. Since $x_{1}+\alpha+\beta \in \Lambda\left(P_{1}\right)$ by assumption, this proves that $\left(x_{1}+\alpha+\beta, x_{2}-\beta-\alpha\right) \in \Lambda\left(P_{1}\right) \times \Lambda\left(P_{2}\right)$. It remains to prove the final assertion. Suppose that $\left(x_{1}+\alpha, x_{2}-\alpha\right) \notin \Lambda\left(P_{1}\right) \times \Lambda\left(P_{2}\right)$. By assumption $x_{1}+\alpha \in \Lambda\left(P_{1}\right)$, so $x_{2}-\alpha \notin \Lambda\left(P_{2}\right)$. In view of Proposition $2.4,\left\langle x_{2}, \alpha^{\vee}\right\rangle \leq 0$. Since $\left\langle x_{2}-\beta, \alpha^{\vee}\right\rangle \geq 1$ (as observed above), this implies that $\left\langle\beta, \alpha^{\vee}\right\rangle \leq-1$. In this case, $\alpha+\beta$ must be a root.

5.4. Proof of Lemma 4.8. First, consider the case that $\left\langle y, \beta^{\vee}\right\rangle<0$. Then we can let $\left(y_{1}, \ldots, y_{m-1}\right) \in \Lambda\left(P_{1}\right) \times \cdots \times \Lambda\left(P_{m-1}\right)$ be arbitrary such that $y=y_{1}+\cdots+y_{m-1}$, and then for some $j$ one must have $\left\langle y_{j}, \beta^{\vee}\right\rangle<0$ as well so that $y_{j}+\beta \in \Lambda\left(P_{j}\right)$ by Proposition 2.4. Similarly, if $\left\langle y, \beta^{\vee}\right\rangle \geq 0$, then $\left\langle y+\beta, \beta^{\vee}\right\rangle>0$, and we can take any $\left(z_{1}, \ldots, z_{m-1}\right) \in \Lambda\left(P_{1}\right) \times \cdots \times \Lambda\left(P_{m-1}\right)$ such that $y+\beta=z_{1}+\cdots+z_{m-1}$. Then there exists some $j$ such that $\left\langle z_{j}, \beta^{\vee}\right\rangle>0$, so again by Proposition $2.4, z_{j}-\beta \in \Lambda\left(P_{j}\right)$. Hence, the tuple $\left(z_{1}, \ldots, z_{j-1}, z_{j}-\beta, z_{j+1}, \ldots, z_{m}\right)$ satisfies the needed conditions. 
6. Ample polytopes are not diagonally split, after Payne. As mentioned in the Introduction, Payne (in [23]) considers a toric variety, $U$, similar to the one we consider, $V$, but for which the rays of the fan are $\mathbb{R}_{\geq 0} \cdot \alpha$, for all $\alpha \in \Phi$. He proves that, in types $A, B, C$ and $D$, for all lattice polytopes $P$ corresponding to a torus-equivariant line bundle on $U$ (even if not ample), the corresponding semi-group $S_{P}$ is normal, and the ring $\mathbb{C}\left[S_{P}\right]$ is Koszul. This follows from the fact, that he proves, that such lattice polytopes are diagonally split for some integer $q \geq 2$.

Here we show that ample polytopes for the varieties $V$ considered in this paper are diagonally split for some integer $q \geq 2$ only in the cases $A_{1}, A_{2}, A_{3}$ and $B_{2}\left(=C_{2}\right)$.

Recall from [23] the following definition. Let $\Gamma$ be a lattice with dual lattice $\Gamma^{\vee}$, and let $\Gamma_{\mathbb{R}}:=\Gamma \otimes_{\mathbb{Z}} \mathbb{R}$ and $\Gamma_{\mathbb{R}}^{\vee}:=\Gamma^{\vee} \otimes_{\mathbb{Z}} \mathbb{R}$. Let $P \subseteq \Gamma \otimes_{\mathbb{Z}} \mathbb{R}$ be a lattice polytope (with vertices in $\Gamma$ ). Let $v_{1}, \ldots, v_{k} \in \Gamma^{\vee}$ be the primitive lattice generators of the inward normal rays of the facets of $P$. Define

$$
\mathbb{F}_{P}^{\circ}:=\left\{u \in \Gamma_{\mathbb{R}} \mid-1<\left\langle u, v_{i}\right\rangle<1, \forall i=1, \ldots, k\right\}
$$

Let $q \geq 2$ be an integer. Then $P$ is diagonally split for $q$ if and only if every element $z \in\left(\frac{1}{q} \bar{\Gamma}\right) / \Gamma$ has a representative $\tilde{z} \in \mathbb{F}_{P}^{\circ} \cap \frac{1}{q} \Gamma$.

Note that, in our case, $\Gamma=Y$. It is clear that all lattice polytopes corresponding to equivariant line bundles on a toric variety are diagonally split if and only if the polytopes corresponding to ample bundles are diagonally split. Moreover, such polytopes are diagonally split if and only if any one such polytope is diagonally split.

Proposition 6.2. An ample polytope (in $Y_{\mathbb{R}}$, with vertices in $Y$ ) is diagonally split for some $q \geq 2$ if and only if the root system is of type $A_{1}, A_{2}, A_{3}$ or $B_{2}\left(=C_{2}\right)$. For $A_{1}$ and $A_{2}$, ample polytopes are diagonally split for all $q \geq 2$, and for $A_{3}$ and $B_{2}$, ample polytopes are diagonally split for odd but not even $q \geq 2$.

Proof. The inward primitive normal vectors for an ample polytope are the images under the Weyl group of the fundamental co-weights $\omega_{i}, i \in I$. Hence, the polytope is diagonally split for $q$ if and only if, for all $z \in \frac{1}{q} Y / Y$, there is a representative $\tilde{z} \in \frac{1}{q} Y$ such that $-1<\left\langle w \tilde{z}, \omega_{i}\right\rangle<1$ for all $i \in I$ and all $w \in W$.

We first prove that such polytopes are not diagonally split if the root system is not listed above. Such root systems contain, as a subsystem, either a root system of type $A_{4}, D_{4}, B_{3}, C_{3}$ or $G_{2}$. It is clear that, for this direction, it suffices to show that, for every $q \geq 2$, ample polytopes for these five root systems are not diagonally split. To do so, it suffices to exhibit in each of these cases a particular element $z \in \frac{1}{q} Y / Y$ such that, for all representatives $\tilde{z} \in \frac{1}{q} Y$, there exists $w \in W$ and $i \in I$ such that $\left|\left\langle w \tilde{z}, \omega_{i}\right\rangle\right| \geq 1$.

We use the standard labelling of roots as in [2, Section VI.4] (which we will also recall). Also, for every $i \in I$, we denote by $s_{i}$ the simple reflection corresponding to the simple root $\alpha_{i}$.

First, let us consider a root system of type $A_{3}$ and even $q$, and show that $P$ is not diagonally split. Recall that, for $A_{n}$ type, the simple roots $\alpha_{1}, \ldots, \alpha_{n}$ are linearly ordered along a line segment. We consider the element $z:=\frac{1}{2} \alpha_{1}+\frac{1}{2} \alpha_{3}$. Then, for every $\tilde{z} \in z+Y$, either $\left|\left\langle\tilde{z}, \omega_{i}\right\rangle\right| \geq 1$ for some $i$, or $\tilde{z}$ is in the same Weyl orbit as $z$. But $\left\langle s_{2} z, \omega_{2}\right\rangle=1$, which yields the desired inequality. In particular, ample polytopes for any root system containing $A_{3}$ are not diagonally split for even $q$. Also, the same is true for root systems containing $B_{3}$ or $C_{3}$. Thus, for the cases $A_{4}, D_{4}, B_{3}$ and $C_{3}$, it suffices to restrict our attention to the case where $q$ is odd. 
From now on, fix an odd integer $q \geq 3$ and set $p:=\frac{q-1}{2}$. Suppose that the root system is of type $A_{4}$. Then we consider the element

$$
z:=\frac{p+1}{q} \alpha_{1}+\frac{p+1}{q} \alpha_{3}+\frac{1}{q} \alpha_{4} .
$$

The only elements $\tilde{z} \in z+\frac{1}{q} Y$ that we need to consider are the eight elements

$$
\tilde{z}=z-\delta_{1} \alpha_{1}-\delta_{3} \alpha_{3}-\delta_{4} \alpha_{4}, \quad \delta_{i} \in\{0,1\} .
$$

First, consider the case that $\left(\delta_{1}, \delta_{3}\right) \neq(1,1)$. If also $\left(\delta_{1}, \delta_{3}\right) \neq(0,0)$, then $\left|\left\langle s_{2} s_{1} \widetilde{z}, \omega_{2}\right\rangle\right|=$ 1. If $\left(\delta_{1}, \delta_{3}\right)=(0,0)$, then $\left\langle s_{2} \widetilde{z}, \omega_{2}\right\rangle=\frac{q+1}{q}>1$.

Next, consider the case that $\delta_{1}=\delta_{3}=1$ and $\delta_{4}=0$. Then $s_{3} \tilde{z}=-\frac{p}{q} \alpha_{1}+\frac{p+1}{q} \alpha_{3}+$ $\frac{1}{q} \alpha_{4}$, which is the case we have already considered in the preceding paragraph.

Thus, it remains to consider the case $\delta_{1}=\delta_{3}=\delta_{4}=1$. Then $s_{4} \tilde{z}=-\frac{p}{q} \alpha_{1}-$ $\frac{p}{q} \alpha_{3}+\frac{p}{q} \alpha_{4}$. Hence, $s_{3} s_{4} \tilde{z}=-\frac{p}{q} \alpha_{1}+\frac{q-1}{q} \alpha_{3}+\frac{p}{q} \alpha_{4}$. Finally, $s_{2} s_{1} s_{3} s_{4} \tilde{z}=\frac{p}{q} \alpha_{1}+\frac{p+q-1}{q} \alpha_{2}+$ $\frac{q-1}{q} \alpha_{3}+\frac{p}{q} \alpha_{4}$, and hence $\left\langle s_{2} s_{1} s_{3} s_{4} \tilde{z}, \omega_{2}\right\rangle \geq 1$.

Hence, ample polytopes for root systems containing $A_{4}$ are not diagonally split for odd $q \geq 3$, and together with the even case above, they are not diagonally split for any $q \geq 2$.

Next, consider the root system $D_{4}$. As in [2, Section VI.4], $\alpha_{2}$ is the simple root corresponding to the node, and $\alpha_{1}, \alpha_{3}$ and $\alpha_{4}$ are the other simple roots. Define the element

$$
z=\frac{p}{q}\left(\alpha_{1}+\alpha_{3}+\alpha_{4}\right)
$$

Similar to the $A_{4}$ case, we only need to consider the elements

$$
\tilde{z}=z-\delta_{1} \alpha_{1}-\delta_{3} \alpha_{3}-\delta_{4} \alpha_{4}, \quad \delta_{i} \in\{0,1\} .
$$

If $\delta_{1}=\delta_{3}=\delta_{4}$ then we see that $\left|\left\langle s_{2} \tilde{z}, \omega_{2}\right\rangle\right| \geq 1$. For the other cases, using symmetry, we may assume that $\delta_{1}=\delta_{3}=0$ and $\delta_{4}=1$. Then $\left|\left\langle s_{2} s_{4} \tilde{z}, \omega_{2}\right\rangle\right|>1$. Hence, ample polytopes containing $D_{4}$ are not diagonally split.

Consider now the root system $B_{3}$, with simple roots $\alpha_{1}, \alpha_{2}, \alpha_{3}$ so that $\alpha_{2}$ corresponds to the central vertex and $\alpha_{3}$ is the short simple root. Let $z=\frac{p}{q}\left(\alpha_{1}+\alpha_{3}\right)$. Then $\left|\left\langle s_{3} s_{2} z, \omega_{3}\right\rangle\right| \geq 1$, and the same is true if we replace $z$ by $z-\left(\alpha_{1}+\alpha_{3}\right), s_{1}\left(z-\alpha_{1}\right)$ or $s_{3}\left(z-\alpha_{3}\right)$. This proves the desired inequality so that ample polytopes containing $B_{3}$ are not diagonally split.

Similarly, consider the root system $C_{3}$ with simple roots $\alpha_{1}, \alpha_{2}, \alpha_{3}$ such that $\alpha_{2}$ corresponds to the central vertex and $\alpha_{3}$ is the long simple root. Let $z:=\frac{p}{q}\left(\alpha_{1}+\alpha_{3}\right)$. Then $\left|\left\langle s_{2} z, \omega_{2}\right\rangle\right| \geq 1$, and the same is true if we replace $z$ by $z-\left(\alpha_{1}+\alpha_{3}\right), s_{1}\left(z-\alpha_{1}\right)$ or $s_{3}\left(z-\alpha_{3}\right)$.

Finally, consider the root system $G_{2}$, and now allow $q \geq 2$ to be any integer. Let $p=\left\lfloor\frac{q}{2}\right\rfloor$. Let $\alpha_{1}$ be the short simple root and $\alpha_{2}$ be the long simple root. Consider $z:=\frac{p}{q} \alpha_{2}$. Then $\left|\left\langle s_{1} z, \omega_{1}\right\rangle\right| \geq 1$. The same is true if we replace $z$ by $s_{2}\left(z-\alpha_{2}\right)$. This proves that ample polytopes are not diagonally split for $G_{2}$.

This completes the proof that ample polytopes for root systems other than $A_{1}, A_{2}, A_{3}$ and $B_{2}$ are not diagonally split for any $q \geq 2$. We also claim that ample 
polytopes are not diagonally split in the case where $q$ is even and the root system is of type $B_{2}$. For this, let $\alpha_{1}$ be the long simple root and $\alpha_{2}$ be the short simple root. Consider the element $z=\frac{1}{2} \alpha_{1}$. Then the same argument as in the case $G_{2}$ applies.

It remains to prove the claims that ample polytopes are diagonally split for odd $q$ in the cases $A_{1}, A_{2}, A_{3}$ and $B_{2}$, and in the case of $A_{1}$ and $A_{2}$, also for even $q$. For the case $A_{1}$, this is clear, and in the case $A_{2}$, it follows by choosing, for any $z \in \frac{1}{q} Y / Y$, the representative $\tilde{z} \in \frac{1}{q} Y$ such that $\left\langle\tilde{z}, \omega_{i}\right\rangle \in[0,1)$ for both fundamental co-weights $\omega_{i}$. Next, consider the case $B_{2}$, and let $q \geq 3$ be odd. Let $\alpha_{1}$ be the long root and $\alpha_{2}$ be the short root. Then, for any $z \in \frac{1}{q} Y / Y$, choose the representative $\tilde{z} \in \frac{1}{q} Y$ such that $\left|\left\langle\tilde{z}, \omega_{1}\right\rangle\right|<\frac{1}{2},\left|\left\langle\tilde{z}, \omega_{2}\right\rangle\right|<1$ and $\left\langle\tilde{z}, \omega_{1}\right\rangle$ and $\left\langle\tilde{z}, \omega_{2}\right\rangle$ are either both non-negative or both non-positive. It is easy to verify that $\tilde{z} \in \mathbb{F}_{P}^{\circ} \cap \frac{1}{q} \Gamma$, as required.

Finally, consider the case $A_{3}$ with $q$ odd. Let $\alpha_{1}, \alpha_{2}$ and $\alpha_{3}$ be the simple roots, with $\alpha_{2}$ corresponding to the central vertex. Then, for any $z \in \frac{1}{q} Y / Y$, first suppose that $\left\langle\tilde{z}, \omega_{2}\right\rangle$ is integral for all representatives $\tilde{z}$ of $z$. In this case, choose the representative $\tilde{z}$ so that $\left\langle\tilde{z}, \omega_{2}\right\rangle=0$ and $\left|\left\langle\tilde{z}, \omega_{i}\right\rangle\right|<\frac{1}{2}$ for $i \in\{1,3\}$. Otherwise, if $\left\langle\tilde{z}, \omega_{2}\right\rangle$ is not integral for any representative $\tilde{z}$ of $z$, choose $\tilde{z}$ such that $\left|\left\langle\tilde{z}, \omega_{i}\right\rangle\right|<1$ for all $i$, either all $\left\langle\tilde{z}, \omega_{i}\right\rangle$ are non-negative or all are non-positive, and such that $\left|\left\langle\tilde{z}, \omega_{1}+\omega_{3}\right\rangle\right| \leq 1$ (where $\alpha_{2}$ corresponds to the central vertex). A straightforward computation verifies that this yields a diagonal splitting.

Appendix A. Sharpening Theorem 1.16 to preserve winning conditions. Here we explain that if one restricts to tuples $\left(x_{1}, \ldots, x_{m}\right) \in \Lambda\left(P_{1}\right) \times \cdots \Lambda\left(P_{m}\right)$ such that the $x_{i}$ are winning, then restricting the equivalence relation $\sim$ and the root moves to these tuples, Theorem 1.16 continues to hold.

Theorem A.1. Suppose that $x \in \Lambda\left(P_{1}+\cdots+P_{m}\right)$ is winning. Then

(i) there exists a tuple $\left(x_{1}, \ldots, x_{m}\right) \in \Lambda\left(P_{1}\right) \times \cdots \times \Lambda\left(P_{m}\right)$ of winning elements such that $x=x_{1}+\cdots+x_{m}$;

(ii) if $\left(x_{1}, \ldots, x_{m}\right),\left(x_{1}^{\prime}, \ldots, x_{m}^{\prime}\right) \in \Lambda\left(P_{1}\right) \times \cdots \times \Lambda\left(P_{m}\right)$ are two tuples of winning elements such that $x_{1}+\cdots+x_{m}=x=x_{1}^{\prime}+\cdots+x_{m}^{\prime}$, then the tuples are related by a sequence of root moves that only pass through tuples of winning elements.

This sharpens the theorem, and further explains its proof.

Remark A.2. Note that, in contrast to Theorem 1.16 itself, even when $P_{1}=\cdots$ $=P_{m}$, it is not necessarily true that all root moves through tuples of winning elements are generated by root moves involving only adjacent indices $j=i+1 \mathrm{in}(1.15)$. This is because adjacent elements in a tuple $\left(x_{1}, \ldots, x_{m}\right)$ of winning elements with $x_{1}+\cdots+$ $x_{m}$ winning need not themselves sum to a winning element. So one cannot deduce that there is a sequence of root moves between adjacent elements that swaps the two elements while only passing through pairs of winning elements.

The theorem rests on the following observation.

Lemma A.3. Suppose that $x \in \Lambda(P)$ is winning. If $\left\langle x, \alpha_{i}^{\vee}\right\rangle \geq 1$, then $x-\alpha_{i} \in \Lambda(P)$ is also winning.

Proof. Suppose that $x \in \Lambda(P)$ is winning and $\left\langle x, \alpha_{i}^{\vee}\right\rangle \geq 1$. First note that $x-\alpha_{i} \in$ $\Lambda(P)$ by Proposition 2.4. Next, if $\left\langle x, \alpha_{i}^{\vee}\right\rangle=1$, then $x-\alpha_{i} \mapsto x$ under the numbers game, so $x-\alpha_{i}$ is also winning. Suppose now that $\left\langle x, \alpha_{i}^{\vee}\right\rangle \geq 2$. By firing vertices other 
than $i$ which are not adjacent to $i$, we may assume that $\left\langle x, \alpha_{j}^{\vee}\right\rangle \geq 0$ whenever $j$ is not adjacent to $i$. Since for $j$ adjacent to $i,\left\langle x, \alpha_{j}^{\vee}\right\rangle \geq-1$, and also $\left\langle\alpha_{i}, \alpha_{j}^{\vee}\right\rangle \leq-1$, it follows that $\left\langle x-\alpha_{i}, \alpha_{j}^{\vee}\right\rangle \geq 0$ for all $j$ adjacent to $i$. Moreover, since $\left\langle\alpha_{i}, \alpha_{i}^{\vee}\right\rangle=2$, it also follows that $\left\langle x-\alpha_{i}, \alpha_{i}^{\vee}\right\rangle \geq 0$. Hence, $x-\alpha_{i}$ is dominant, and therefore winning. (Without assuming that $\left\langle x, \alpha_{j}^{\vee}\right\rangle \geq 0$ whenever $j$ is not adjacent to $i$, we then see that $x-\alpha_{i}$ will be winning, but not dominant in general.)

\section{A.1. Simple moves.}

Definition A.4. Let $\left(x_{1}, \ldots, x_{m}\right) \in X^{m}$. Suppose that $\alpha$ is a simple root and $j, k$ are indices such that $\left\langle x_{j}, \alpha^{\vee}\right\rangle \leq-1$ and $\left\langle x_{k}, \alpha^{\vee}\right\rangle \geq 1$. Then setting $x_{j}^{\prime}=x_{j}+\alpha$ and $x_{k}=x_{k}-\alpha$, and $x_{\ell}^{\prime}=x_{\ell}$ for $\ell \notin\{j, k\}$, we say $\left(x_{1}^{\prime}, \ldots, x_{m}^{\prime}\right)$ is obtained from $\left(x_{1}, \ldots x_{m}\right)$ by a simple move.

Note that a simple move is a very special type of root move, in the case that all the elements of the tuples are in the relevant polytopes. In fact, it is enough to check this for one of the tuples.

LemmA A.5. If $P_{1}, \ldots, P_{m}$ are special ample polytopes, $\left(x_{1}, \ldots, x_{m}\right) \in \Lambda\left(P_{1}\right) \times \cdots$ $\times \Lambda\left(P_{m}\right)$, and $\left(x_{1}^{\prime}, \ldots, x_{m}^{\prime}\right)$ is obtained from $\left(x_{1}, \ldots, x_{m}\right)$ by simple moves, then also $\left(x_{1}^{\prime}, \ldots, x_{m}^{\prime}\right) \in \Lambda\left(P_{1}\right) \times \cdots \times \Lambda\left(P_{m}\right)$.

The lemma is an immediate consequence of Proposition 2.4. Furthermore, using Lemma A.3, we can prove the following.

LemmA A.6. If $\left(x_{1}, \ldots, x_{m}\right) \in \Lambda\left(P_{1}\right) \times \cdots \times \Lambda\left(P_{m}\right)$ is a tuple of winning elements, then any simple move results in another tuple of winning elements.

Proof. It is clear enough to assume $m=2$. Without loss of generality, the move is $\left(x_{1}, x_{2}\right) \mapsto\left(x_{1}+\alpha, x_{2}-\alpha\right)$ where $\left\langle x_{1}, \alpha^{\vee}\right\rangle<0$ and $\left\langle x_{2}, \alpha^{\vee}\right\rangle>0$. Since $x_{1}$ is winning, $\left\langle x_{1}, \alpha^{\vee}\right\rangle=-1$ and $x_{1}+\alpha$ is obtained by playing a move of the numbers game. Hence, $x_{1}+\alpha$ is winning. Also, $x_{2}-\alpha$ is winning by Lemma A.3.

Also, the proof of Lemma 4.6 actually implies.

Lemma A.7. Let $P_{1}$ and $P_{2}$ be special ample polytopes, $\left(x_{1}, x_{2}\right) \in \Lambda\left(P_{1}\right) \times \Lambda\left(P_{2}\right)$, $P=P_{1}+P_{2}$ and $x=x_{1}+x_{2} \in \Lambda(P)$. If $\alpha$ is $P$-progressive for $x$, then there is a sequence of simple moves taking $\left(x_{1}, x_{2}\right)$ to a pair $\left(x_{1}^{\prime}, x_{2}^{\prime}\right)$ such that, for some $i \in\{1,2\}, \alpha$ is $P_{i^{-}}$ progressive for $x_{i}^{\prime}$.

Hence, if $\left(x_{1}, x_{2}\right)$ additionally has the property that $x_{1}$ and $x_{2}$ are winning, Lemma A.6 implies that all pairs obtained along the way from $\left(x_{1}, x_{2}\right)$ to $\left(x_{1}^{\prime}, x_{2}^{\prime}\right)$ (along with $\left(x_{1}^{\prime}, x_{2}^{\prime}\right)$ itself) consist of winning elements.

A.2. The case $m=2$ of Theorem A.1.(ii). In order to explain the general result, it is best to begin with the case $m=2$.

Proof of Theorem A.1.(ii) for $m=2$. Without the winning conditions, this is the case $m=2$ of Theorem 1.16.(ii). Let $x=x_{1}+x_{2}$ and let $\alpha$ be $P$-progressive for $x$. We assume the statement for tuples of winning elements which sum to $x+\alpha$. In the proof of Theorem 1.16.(ii), the moves taken are either simple, which preserve the property of elements being winning by Lemma A.6, or else are moves obtained by Lemma 4.7 from root moves for a tuple whose sum is $x+\alpha$. As in the proof of Theorem 1.16.(ii), 
the first such move of the latter type begins with a tuple $\left(x_{1}, x_{2}\right)$ of winning elements such that $\alpha$ is $P_{1}$-progressive for $x_{1}$. By Proposition 4.3, then $x_{1}+\alpha$ is winning and in $\Lambda\left(P_{1}\right)$. By induction on the number of such moves required, we can then assume that the latter root move is of the form $\left(x_{1}+\alpha, x_{2}\right) \mapsto\left(x_{1}+\alpha+\beta, x_{2}-\beta\right)$ for $\beta \in \Phi$ such that $x_{1}+\alpha+\beta$ and $x_{2}-\beta$ are winning. Thus, it remains to prove the following sharpening of Lemma 4.7.

LemmA A.8. Suppose that $\left(x_{1}, x_{2}\right) \in \Lambda\left(P_{1}\right) \times \Lambda\left(P_{2}\right), x_{1}$ and $x_{2}$ are winning, and $\alpha$ is a simple root such that $x_{1}+\alpha$ is winning and in $\Lambda\left(P_{1}\right)$, and $x=x_{1}+x_{2}$ satisfies $\left\langle x, \alpha^{\vee}\right\rangle \geq-1$. If $\beta \in \Phi$ is such that $\left(x_{1}+\alpha+\beta, x_{2}-\beta\right) \in \Lambda\left(P_{1}\right) \times \Lambda\left(P_{2}\right)$ is a tuple of winning elements, then either $\left(x_{1}+\beta, x_{2}-\beta\right) \in \Lambda\left(P_{1}\right) \times \Lambda\left(P_{2}\right)$ or $\left(x_{1}+\alpha+\beta, x_{2}-\right.$ $\alpha-\beta) \in \Lambda\left(P_{1}\right) \times \Lambda\left(P_{2}\right)$, and it is a pair of winning elements. Furthermore, in the latter case, either $\left(x_{1}+\alpha, x_{2}-\alpha\right)$ is in $\Lambda\left(P_{1}\right) \times \Lambda\left(P_{2}\right)$ and is a pair of winning elements, or else $\alpha+\beta \in \Phi$.

Proof. To prove the first assertion, we only need to check that, following the proof of Lemma 4.7, the final tuple $\left(x_{1}+\beta, x_{2}-\beta\right)$ or $\left(x_{1}+\alpha+\beta, x_{2}-\alpha-\beta\right)$ is a tuple of winning elements. In the first case, we had $\left\langle x_{1}+\alpha+\beta, \alpha^{\vee}\right\rangle \geq 1$, and $x_{1}+$ $\alpha+\beta$ is winning, so Lemma A.3 implies that $x_{1}+\beta$ is winning; $x_{2}-\beta$ is winning by assumption. In the second case, we had $\left\langle x_{2}-\beta, \alpha^{\vee}\right\rangle \geq 1$ and $x_{2}-\beta$ is winning, and hence $x_{2}-\beta-\alpha$ is winning by Lemma A. $3 ; x_{1}+\alpha+\beta$ is winning by assumption. For the final statement, we note that if $\left(x_{1}+\alpha, x_{2}-\alpha\right)$ is not winning, then in view of Lemma A.3 $\left\langle x_{2}, \alpha^{\vee}\right\rangle \leq 0$, and the statement then follows as in the proof of Lemma 4.7 .

A.3. Proof for general $m$. As in the proof of Theorem 1.16, let $P=P_{1}+\cdots+P_{m}$ and $\alpha$ be a simple root that is $P$-progressive for $x$.

(i) This follows from the proof of Theorem 1.16.(i). We note that if $\left(y_{1}, \ldots, y_{m}\right)$ is a tuple of winning elements with $y_{1}+\cdots y_{m}=x+\alpha$ and $\left\langle y_{i}, \alpha^{\vee}\right\rangle \geq 1$, then $y_{i}-\alpha$ is still winning by Lemma A.3, and hence $\left(y_{1}, \ldots, y_{i-1}, y_{i}-\alpha, y_{i+1}, \ldots, y_{m}\right)$ is a tuple of winning elements summing to $x$.

(ii) We adapt the proof of Theorem 1.16.(ii). Let $Q:=P_{1}+\cdots+P_{m-1}, y=$ $x_{1}+\cdots+x_{m-1}$, and $y^{\prime}=x_{1}^{\prime}+\cdots+x_{m-1}^{\prime}$. We assume the statement of the theorem for tuples whose sum is $x$. The proof below will be slightly more complicated than the proof of Theorem 1.16.(ii), because we cannot in general assume that $y$ is winning, and hence cannot apply the statement of the theorem to $y$ itself (and in particular, we do not need to assume the statement of the theorem for smaller values of $m$ ).

By performing simple moves, we can assume that $\alpha$ is either $Q$-progressive for $y$ or $P_{m}$-progressive for $x_{m}$. In the case it is $Q$-progressive for $y$, we can iterate this procedure on the tuple $\left(x_{1}, \ldots, x_{m-1}\right)$ until there exists an index $i$ such that $\alpha$ is $P_{i}$-progressive for $x_{i}$. So we assume this. By doing the same for $\left(x_{1}^{\prime}, \ldots, x_{m}^{\prime}\right)$, we can suppose that $\alpha$ is $P_{j}$-progressive for $x_{j}^{\prime}$. By hypothesis, $\left(x_{1}, \ldots, x_{i-1}, x_{i}+\alpha, x_{i+1}, \ldots, x_{m}\right)$ and $\left(x_{1}^{\prime}, \ldots, x_{j-1}^{\prime}, x_{j}^{\prime}+\alpha, x_{j+1}^{\prime}, \ldots, x_{m}^{\prime}\right)$ are related by root moves which pass only through winning tuples (since these are tuples whose sum is $x+\alpha$ ).

It is then enough to show that for a single root move $\left(x_{1}, \ldots, x_{i-1}, x_{i}+\right.$ $\left.\alpha, x_{i+1}, \ldots, x_{m}\right) \mapsto\left(y_{1}, \ldots, y_{m}\right)$ (with $x_{\ell}, y_{\ell} \in \Lambda\left(P_{\ell}\right)$ winning for all $\ell$, and $x_{i}+\alpha \in \Lambda\left(P_{i}\right)$ winning), there exists an index $k$ such that $y_{k}-\alpha$ is in $\Lambda\left(P_{k}\right)$ and winning, and such that $\left(x_{1}, \ldots, x_{m}\right)$ is related to $\left(y_{1}, \ldots, y_{k-1}, y_{k}-\alpha, y_{k+1}, \ldots, y_{m}\right)$ by root moves that pass only through tuples of winning elements. If there exists an 
index $k$ such that $x_{k}=y_{k}$ and $\left\langle x_{k}, \alpha^{\vee}\right\rangle \geq 1$, then the statement follows immediately. If not, then the root move is of the form $\left(x_{i}+\alpha, x_{j}\right) \mapsto\left(x_{i}+\alpha+\beta, x_{j}-\beta\right)$ for some $\beta \in \Phi$, and $\left\langle x_{\ell}, \alpha^{\vee}\right\rangle \leq 0$ for all $\ell \notin\{i, j\}$. Since $\left\langle x+\alpha, \alpha^{\vee}\right\rangle \geq 1$ (as $\alpha$ is $P$-progressive for $x)$, it follows that $\left\langle\left(x_{i}+\alpha\right)+x_{j}, \alpha^{\vee}\right\rangle \geq 1$, and hence $\left\langle x_{i}+x_{j}, \alpha^{\vee}\right\rangle \geq-1$. Now the statement follows from Lemma A.8 (applied to the pair $\left(x_{i}, x_{j}\right)$ together with $\alpha$ and $\beta) ; k$ will then be either $i$ or $j$.

ACKNOWLEDGEMENTS. We thank Sam Payne and the anonymous referee for helpful comments. The first author was supported by an EPDI Fellowship. The second author is an AIM Five-Year Fellow, and was partially supported by the ARRA-funded NSF grant DMS-0900233. We thank IHES and MIT for hospitality.

\section{REFERENCES}

1. J. Arthur, A local trace formula, Inst. Hautes Études Sci. Publ. Math. 73 (1991), 5-96.

2. N. Bourbaki, Elements of mathematics, Lie groups and Lie algebras, chapters 4-6, (Springer-Verlag, Berlin, Germany, 2002).

3. J. Carrell and A. Kurth, Normality of torus orbit closures in $G / P$, J. Algebra 233(1) (2000), 122-134.

4. J. Carrell and J. Kuttler, Smooth points of $t$-stable varieties in $G / B$ and the Peterson map, Invent. Math. 151(2) (2003), 353-379.

5. R. Dabrowski, On normality of the closure of a generic torus orbit in $G / P$, Pacific $J$. Math. 172(2) (1996), 321-330.

6. R. G. Donnelly and K. Eriksson, The numbers game and Dynkin diagram classification results (2008) arXiv:0810.5371.

7. K. Eriksson, Convergence of Mozes's game of numbers, Linear Algebra Appl. 166 (1992), 151-165.

8. K. Eriksson, Strongly convergent games and Coxeter groups, $\mathrm{PhD}$ thesis, (KTH, Stockholm, Sweden, 1993).

9. K. Eriksson, Node firing games on graphs, Jerusalem combinatorics '93: an international conference in combinatorics (May 9-17, 1993, Jerusalem, Israel), vol. 178 (American Mathematical Society, 1994), 117-128.

10. K. Eriksson, Reachability is decidable in the numbers game, Theoret. Comput. Sci. 131 (1994), 431-439. 166.

11. K. Eriksson, The numbers game and Coxeter groups, Discrete Math 139 (1995), 155

12. K. Eriksson, Strong convergence and a game of numbers, Eur. J. Combin. 17(4) (1996), 379-390.

13. W. Fulton, Introduction to toric varieties, Annals of Mathematics Studies, no.131 (Princeton University Press, Princeton, NJ, 1993).

14. Q. R. Gashi, A vanishing result for toric varieties associated with root systems, Albanian J. Math. 4 (2007), 235-244.

15. Q. R. Gashi, The conjecture of Kottwitz and Rapoport in the case of split groups, PhD thesis, (The University of Chicago, Chicago, IL, June 2008).

16. Q. R. Gashi, The Conjecture of Kottwitz and Rapoport in the case of split groups (2008); arXiv:0805.4575.

17. Q. R. Gashi, Vanishing results for toric varieties associated to $G L_{n}$ and $G_{2}$, Transform. Groups 13(1) (2008), 149-171.

18. Q. R. Gashi and T. Schedler, On dominance and minuscule Weyl group elements J. Algebraic Combin. 33(3) (2011), 383-399.

19. Benjamin. J. Howard, Matroids and geometric invariant theory of torus actions on flag spaces, J. Algebra 312(1) (2007), 527-541.

20. A. Klyachko, Toric varieties and flag spaces, Teor. Chisel, Algebra i Algebr. Geom., no. 208 (Trudy Mat. Inst. Steklova, Moscow, Russia, 1995), 139-162. 
21. R. E. Kottwitz, Harmonic analysis on reductive $p$-adic groups and Lie algebras, in Harmonic analysis, the trace formula, and Shimura varieties (J. Arthur et al., Editors), Clay Math. Proc., no. 4 (American Mathematical Society, Providence, RI, 2005), 393-522.

22. S. Mozes, Reflection processes on graphs and Weyl groups, J. Combin. Theory Ser. A 53(1) (1990), 128-142.

23. S. Payne, Lattice polytopes cut out by root systems and the Koszul property, Adv. Math. 220(3) (2009), 926-935.

24. C. Procesi, The toric variety associated to Weyl chambers (English summary) Mots (Lang. Raison. Calc., Hermès, Paris, France, 1990), 153-161.

25. R. A. Proctor, Bruhat lattices, plane partition generating functions, and minuscule representations, Euro. J. Combin. 5 (1984), 331-350.

26. R. A. Proctor, Minuscule elements of Weyl groups, the numbers game, and $d$-complete posets, J. Algebra 213 (1999), 272-303.

27. M. Rapoport, A positivity property of the Satake isomorphism, Manuscripta Math. 101(2) (2000), 153-166. 364.

28. J. R. Stembridge, The partial order of dominant weights, Adv. Math. 136 (1998), 340-

29. B. Sturmfels, Gröbner bases and convex polytopes, University Lecture Series, vol. 8, (American Mathematical Society, Providence, RI, 1996).

30. N. J. Wildberger, A combinatorial construction for simply-laced Lie algebras, $A d v$. in Appl. Math. 30 (2003), 385-396.

31. N. J. Wildberger, Minuscule posets from neighbourly graph sequences, Euro. J. Combin. 24 (2003), 741-757. 